\title{
Two-dimensional substitution: towards a better understanding of the structure-transport correlations in the Li-superionic thio-LISICONs
}

Nicolò Minafra, ${ }^{a}$ Katharina Hogrefe,${ }^{b}$ Federico Barbon, ${ }^{c}$ Bianca Helm,${ }^{d}$ Cheng Li, ${ }^{e, f}$ H. Martin R. Wilkening, ${ }^{b}$ and Wolfgang G. Zeier*a

${ }^{a}$ Institute of Inorganic and Analytical Chemistry, University of Münster, Correnstrasse 30, 48149 Münster, Germany.

${ }^{b}$ Institute for Chemistry and Technology of Materials, Graz University of Technology, Stremayrgasse 9, A-8010 Graz, Austria.

${ }^{c}$ Dipartimento di Scienze Chimiche, Università Degli Studi di Padova, Via Marzolo 1, 35131, Padova, Italy.

${ }^{d}$ Institute of Physical Chemistry, Justus-Liebig-University Giessen, Heinrich-Buff-Ring 17, D-35392 Giessen, Germany.

e Jülich Centre for Neutron Science (JCNS), Forschungszentrum Jülich GmbH, Outstation at SNS, 1 Bethel Valley Road, Oak Ridge, Tennessee 37831-6473, United States.

${ }^{f}$ Neutron Scattering Division, Oak Ridge National Laboratory (ORNL), 1 Bethel Valley Road, Oak Ridge, Tennessee 37831-6473, United States.

*wzeier@uni-muenster.de 


\begin{abstract}
A deeper understanding of the relationships among composition-structure-transport properties in inorganic solid ionic conductors is of paramount importance to develop highly conductive phases for future employment in solid-state Li-ion battery applications. In order to shed light on the mechanisms that regulate these relationships, in this work we perform a "twodimensional" substitution series in the thio-LISICON family $\mathrm{Li}_{4} \mathrm{Ge}_{1-x} \mathrm{Sn}_{x} \mathrm{~S}_{4-y} \mathrm{Se}_{y}$. The structural modifications brought up by the elemental substitutions were investigated via Rietveld refinements against high-resolution neutron diffraction data that allowed a precise characterization of the anionic framework and the lithium substructure. The analyses show that the anionic and cationic substitutions influence the polyhedral and unit cell volumes in different fashions and that the size of the polyanionic groups alone is not enough to describe lattice expansion in these materials. Moreover, we show that the lithium disorder that is crucial to achieve fast ionic mobility may be correlated to the lithium polyhedral volumes. The correlation of these structural modifications with the transport properties, investigated via electrochemical impedance spectroscopy and ${ }^{7} \mathrm{Li}$ nuclear magnetic resonance spin-lattice relaxation measurements, shows a non-monotonic behavior of the ionic conductivity and activation energy against the lithium polyhedral volumes, hinting to an optimal size of the conduction pathways for the ionic diffusion. Ultimately, the results obtained in this work will help to establish new guidelines for the optimization of solid electrolytes and to gain a more profound understanding of the influence of the substituents on the structure and transport properties of Li-ion conductors.
\end{abstract}




\section{Introduction}

For a further improvement of lithium-ion batteries, the employment of a solid material as the electrolyte may enhance the electrochemical performance and improve the device safety of the current cell architectures.$^{1-3}$ In order to guarantee an optimal cell operation, the solid electrolyte, among other requirements, must allow fast $\mathrm{Li}^{+}$ionic motion among the electrodes. ${ }^{4-6}$ While many highly conducting phases have been recently synthesized, ${ }^{7-11}$ the list of materials that exhibit high ionic conductivity at room temperature is still extremely short due to the strict structural conditions that need to be satisfied to allow fast ionic motion. ${ }^{12-14}$ High carrier concentration and a disordered lithium substructure are of paramount importance to achieve a high ionic conductivity. ${ }^{15,16}$ Moreover, polyhedral connectivity and different lithium coordination strongly influence the energy landscape for the ionic diffusion, where larger changes of coordination environment lead to higher activation barriers. ${ }^{12}$ Fast ionic jumps are possible via face-sharing tetrahedra, while ionic motion through edge-sharing polyhedra is unfavorable at typical lattice volumes of sulfides and oxides. ${ }^{12,17}$ The anionic framework should also provide wide channels for the ionic motion, leading to the conception that larger lattice volumes are beneficial for the ionic diffusion. ${ }^{17-19}$ However, while this condition seems to be generally valid, a number of studies report on an optimal channel size for the ionic conductivity. ${ }^{20-24}$ After exceeding an optimal value, transport properties either decrease or reach a plateau for larger volumes..$^{20-24}$ In addition to these static structural parameters, a larger anion polarizability is also required to lower the activation energy by means of a weaker cation-anion interaction. ${ }^{25-28}$ All these aspects are highly convoluted as, for instance, more polarizable anionic frameworks often possess larger volumes. Therefore, it is challenging to discern the precise effect that each of these features have on the transport properties.

To date, there are only few classes of solid material that meet the above mentioned conditions, and induce structural modifications via elemental substitutions is a commonly adopted strategy to enhance the ionic conductivity of these already promising phases. ${ }^{12,14,29}$ However, "linear substitutions", i.e. substitutions of one single element in a given structure, do not allow for a deeper understanding of the properties that govern the structure-transport relations of complex crystallographic framework and compositions. Therefore, in order to gain a better understanding of the substituents influence on the structural modification and transport properties, we performed a systematic study of a "two-dimensional" substitution series, i.e. substitutions of cationic and anionic species, in the thio-LISICON family (Figure 1a). 
a)

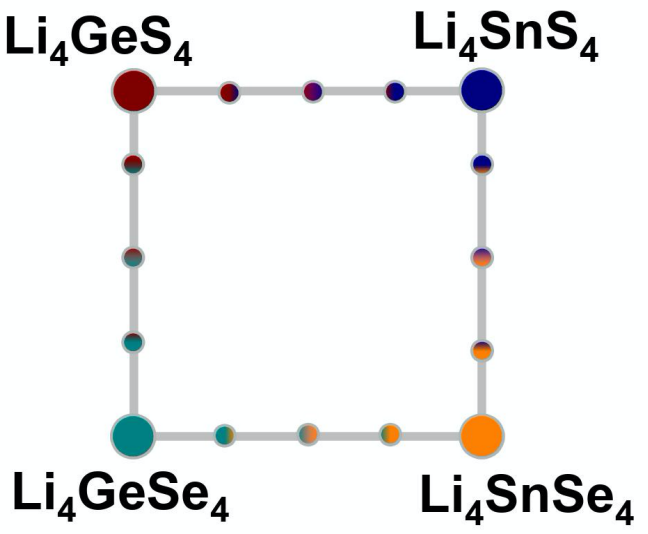

b)

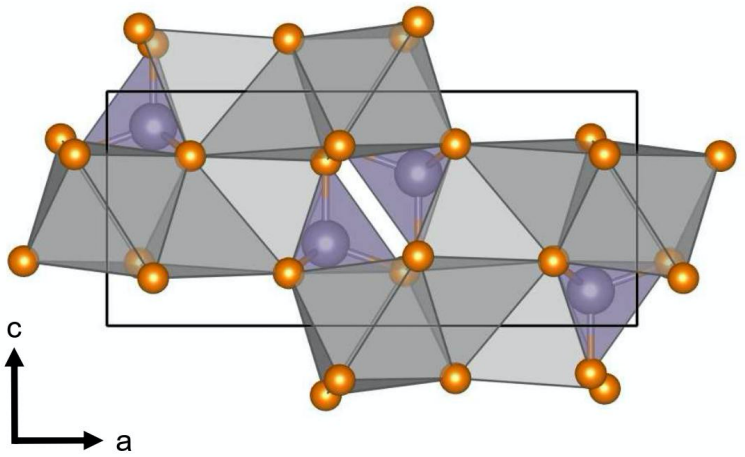

Figure 1: a) Graphic representation of the compositions in the solid solution between $\mathrm{Li}_{4} \mathrm{GeS}_{4}$, $\mathrm{Li}_{4} \mathrm{SnS}_{4}, \mathrm{Li}_{4} \mathrm{GeSe}_{4}$ and $\mathrm{Li}_{4} \mathrm{SnSe}_{4}$ explored in this study. b) Crystal structure of $\mathrm{Li}_{4} \mathrm{SnS}_{4}$ as an example, showing the framework composed of isolated $M S_{4}$ tetrahedra (purple) and a variety of different $\mathrm{Li}-\mathrm{S}$ coordination environments (gray). $\mathrm{Li}^{+}$is omitted for clarity and shown completely in Figures 5 and 6.

The thio-LISICONs are a derivative of the oxide-based LISICON materials composed of a softer and more polarizable sulfide anionic sublattice. ${ }^{30}$ These materials are generally represented by the unit formula $\mathrm{Li}_{x} M \mathrm{~S}_{4}$, where the lithium content depends on the valence of the $M$ cation. An exemplary structure is shown in Figure 1b. The anionic framework of these materials is generally composed of isolated polyanionic $M C h_{4}$ groups whose three-dimensional arrangement depends on the temperature and compositions. ${ }^{31,32}$ At room temperature, these moieties form an hexagonal close packed ( $h c p$ ) anionic sublattice that offers a large number of octahedral and tetrahedral voids suitable for lithium occupation. ${ }^{33}$ The different possible lithium substructures will be discussed in more detail below. Depending on the composition, the lithium ions occupy different sets of positions in a more or less disordered fashion. ${ }^{12}$ While the origin of these differences is still unknown, the different lithium distributions have been shown to strongly impact the transport properties of these materials. ${ }^{12,32,34}$

In order to improve the ionic transport many aliovalent substitutions of the central element in $\mathrm{Li}_{4} M \mathrm{~S}_{4}(M=\mathrm{Si}, \mathrm{Ge}, \mathrm{Sn})^{33-38}$ have been performed. Both substitutions of trivalent elements (i.e. $\mathrm{Ga}, \mathrm{Al})^{30,39-41}$ and pentavalent elements (i.e. $\left.\mathrm{P}, \mathrm{As} \mathrm{Sb}\right)^{39,42-47}$ for $M^{4+}$ result in beneficial transport properties of $\mathrm{Li}_{4} M \mathrm{~S}_{4}$, with the ionic conductivity of the solid solutions being orders of magnitude higher than the end members. This behavior indicates that the structural changes in the compounds with mixed compositions (for both lithium excess or deficient solid solution of $\left.\mathrm{Li}_{4} M \mathrm{~S}_{4}\right)$ strongly affect the transport properties of these materials rather than the carrier concentration per se. ${ }^{12}$ Among others, the $\mathrm{Li}_{4-x} \mathrm{Ge}_{1-x} \mathrm{P}_{x} \mathrm{~S}_{4}$ solid solution results in the highest 
ionic conductivity of these solid electrolytes $\left(\text { i.e. } \sim 2 \cdot 10^{-3} \mathrm{~S} / \mathrm{cm}\right)^{42}$ which led to the discovery of the $\mathrm{Li}_{10} \mathrm{GeP}_{2} \mathrm{~S}_{12}$ phases that show even faster $\mathrm{Li}^{+}$transport. ${ }^{48}$

While cationic substitutions have been broadly studied, anionic substitutions have not been widely explored and only few studies, mainly focused on $\mathrm{Li}_{3} \mathrm{PS}_{4-x} \mathrm{O}_{x}$, can be found in the literature. ${ }^{49-52}$ Apart from the oxygen substitution, a selenide-based material, $\mathrm{Li}_{4} \mathrm{SnSe}_{4},{ }^{53}$ has been synthesized, possessing an ordered lithium substructure and, therefore, lower ionic conductivity compared to the lithium-disordered $\mathrm{Li}_{4} \mathrm{SnS}_{4}$ parent phase..$^{35}$

The thio-LISICON family was chosen as model due to its great structural versatility that allows anionic and cationic elemental substitutions while retaining the main structural identity, namely the polyanionic framework. Therefore, isovalent anionic and cationic substitutions were performed within $\mathrm{Li}_{4} \mathrm{Ge}_{1-x} \mathrm{Sn}_{x} \mathrm{~S}_{4-y} \mathrm{Se}_{y}$ in order to discern the influence of the different chemical nature of the substituents on the structural modifications and their impact on the ionic transport. The structural modifications, investigated by means of Rietveld refinements against highresolution neutron diffraction data, show that the anionic and cationic substitutions have a different influence on the $M C h_{4}$ and lithium polyhedral volume that in turn affect the relative lattice expansion. The substituents also strongly affect the lithium distribution whose ordered or disordered character may be correlated to the lithium polyhedral size. The correlation of these structural modifications with the transport properties, investigated by electrochemical impedance spectroscopy and variable-temperature ${ }^{7}$ Li nuclear magnetic resonance (NMR) spinlattice relaxation measurements, shows a non-monotonic behavior of the ionic conductivity and activation energy against the lithium polyhedral volumes, hinting to an optimal size of the conduction pathways. Ultimately, this work provides new insights on the compositionstructure-transport relationships in solid electrolytes.

\section{Experimental Section}

Synthesis. All preparations and sample treatments were carried out under Ar atmosphere. Initially, lithium selenide was prepared via gas-phase reaction to use as precursor for the final products. Metallic lithium (Li, Alfa-Aesar, 99.9\%) was placed into a graphite crucible which was then inserted into a quartz ampule containing Se powder (Se, Alfa-Aesar, 99.999\%) at the bottom. Prior to the filling, the quartz tube and the carbon crucible were heated at $1073 \mathrm{~K}$ under dynamic vacuum to remove moisture. The reaction was performed at $673 \mathrm{~K}$ for $48 \mathrm{~h}(285 \mathrm{~K} / \mathrm{h}$ heating rate). The obtained product was pulverized and phase purity was confirmed by X-ray powder diffraction. $\mathrm{Li}_{4} M C h_{4}(M=\mathrm{Ge}, \mathrm{Sn} ; C h=\mathrm{S}, \mathrm{Se})$ samples were prepared via high temperature solid-state reaction. Lithium selenide, lithium sulfide ( $\left.\mathrm{Li}_{2} \mathrm{~S}, \mathrm{Alfa}-\mathrm{Aesar}, 99.9 \%\right)$, 
germanium (Ge, ChemPur, 99.995\%), tin (Sn, ChemPur, 99.999\%), sulfur (S, Acros Organics, 99.999\%) and selenium (Se, Alfa-Aesar, 99.999\%) were mixed in the appropriate stoichiometric ratio and hand-ground in an agate mortar for $15 \mathrm{~min}$. The obtained mixtures were pelletized and then filled into quartz ampoules, which were sealed under vacuum. Prior to the filling, the quartz tubes were carbon coated and preheated at $1073 \mathrm{~K}$ under dynamic vacuum to remove all trace of water in the reaction atmosphere. The reactions were performed at $973 \mathrm{~K}$ for 16 hours, and at $873 \mathrm{~K}$ for 48 hours for Ge and Sn containing sample, respectively. After annealing, the obtained products were ground and prepared for the specific characterization method.

Neutron powder diffraction. Neutron powder diffraction data of $\mathrm{Li}_{4} M \mathrm{~S}_{4-y} \mathrm{Se}_{y}(M=\mathrm{Ge}, \mathrm{Sn} ; 0<$ $y \leq 4)$ and $\mathrm{Li}_{4} \mathrm{Ge}_{1-x} \mathrm{Sn}_{x} \mathrm{Se}_{4}(0<x<1)$ were collected at Oak Ridge spallation neutron source (SNS, Oak Ridge National Laboratory) using the PAC automatic sample changer at POWGEN diffractometer (BM11-A beamline).$^{54}$ Approximately $3 \mathrm{~g}$ of sample were loaded into an $8 \mathrm{~mm}$ diameter cylindrical vanadium can under inert atmosphere and sealed with a copper gasket to avoid air exposure during measurements. Using a single bank with center wavelength of $1.5 \AA$, data were collected in high-resolution mode at room temperature for approximately $3 \mathrm{~h}$. This bank allowed to probe a $d$-spacing range from 0.5 up to $6 \AA$ with a resolution $\delta d / d<9 \cdot 10^{-3}$. The obtained diffractograms are shown in the Supporting Information, Figure S1. For the $\mathrm{Li}_{4} \mathrm{Ge}_{1-x} \mathrm{Sn}_{x} \mathrm{~S}_{4}$ series, we refer the reader to reference 34 .

Rietveld analysis. Rietveld refinements were carried out using the TOPAS-Academic V6 software package. ${ }^{55}$ The structural data obtained from neutron refinements of $\mathrm{Li}_{4} M \mathrm{~S}_{4}(M=\mathrm{Ge}$, Sn) from Minafra et al. were used as starting model. ${ }^{34}$ The peak profile shape was described by a convolution of pseudo-Voigt and GSAS back-back exponential function. ${ }^{54}$ Fit indicators $\mathrm{R}_{\mathrm{wp}}$, and Goodness-of-fit (GoF) were used to assess the quality of the refined structural model. The following parameters were initially refined: (1) scale factor, (2) background, (3) peak shape and (4) lattice parameters. After a good fit of the profile was achieved, the structural parameters were allowed to refine. Initially, (5) fractional atomic coordinates, (6) atomic occupancies and (7) isotropic atomic displacement parameters were refined. Finally, lithium occupancy on other possible interstitial sites was investigated. The coordinates of these voids are tabulated in the Supporting Information, Table S1. The stability of the refinements was ensured by allowing to refine multiple correlated parameters simultaneously over several cycles. Finally, the crystallographic information files reported in the Supporting Information were obtained by allowing to refine all possible structural parameters at the same time, further proving the 
stability of the refined structure. The structural data relative to $\mathrm{Li}_{4} \mathrm{Ge}_{1-x} \mathrm{Sn}_{x} \mathrm{~S}_{4}$ were taken from our previous report. ${ }^{34}$

Electrochemical impedance spectroscopy. Electrical conductivities were measured by ac impedance spectroscopy, using isostatically pressed pellets (325 MPa, geometric density of all samples $>80 \%)$ that were subsequently coated via thermal evaporation with thin gold $(200 \mathrm{~nm})$ electrodes. Electrochemical impedance analysis was conducted in the temperature range of 283-333 K using a SP300 impedance analyzer (Biologic) at frequencies from $7 \mathrm{MHz}$ to 50 $\mathrm{mHz}$ with an amplitude of $10 \mathrm{mV}$. The error associate with the results of the impedance measurements (i.e. ionic conductivities and activation energies) was carried out based on standard deviations of triplicates reported in our recent report on the $\mathrm{Li}_{4} \mathrm{Ge}_{1-x} \mathrm{Sn}_{x} \mathrm{~S}_{4}$ solid solution. ${ }^{34}$ The impedance data relative to $\mathrm{Li}_{4} \mathrm{Ge}_{1-x} \mathrm{Sn}_{x} \mathrm{~S}_{4}(0<x<1)$ were taken from our previous study. ${ }^{34}$

Nuclear Magnetic Relaxation. All samples were fire sealed in Duran` glass tubes under vacuum to protect them from any reaction with air or moisture. ${ }^{7} \mathrm{Li}$ NMR spin-lattice relaxation measurements were recorded in both the laboratory and the rotating frame of reference on a Bruker Avance III spectrometer connected to a shimmed cryo magnet with a nominal magnetic field of $7 \mathrm{~T}$. This external magnetic field corresponds to a Larmor frequency of $116 \mathrm{MHz}$. All measurements were carried out in the temperature range of 173 to $333 \mathrm{~K}$ with a ceramic hightemperature probe (Bruker). Experiments to obtain the ${ }^{7} \mathrm{Li}$ NMR spin-lattice relaxation rate in the laboratory frame $\left(1 / T_{1}\right)$ were conducted via the well-known saturation pulse sequence. ${ }^{56}$ For the $\pi / 2$ pulses a power level of $200 \mathrm{~W}$ was chosen; pulse lengths ranged from 2.13 to $2.83 \mu \mathrm{s}$ depending on temperature. The area under the free induction decays was plotted versus the waiting time $t_{\mathrm{d}}$ to construct the magnetization transients $M_{\mathrm{z}}\left(t_{\mathrm{d}}\right)$, which were then analyzed with stretched exponentials containing characterized by the stretching exponent $\gamma$. To record diffusion-controlled spin-lattice relaxation NMR in the rotating frame of reference we employed the classical two-pulse spin-lock pulse sequence, ${ }^{56}$ that allows observing the decay of transversal magnetization $M_{\rho}\left(t_{\text {lock }}\right)$ as a function of the duration of the locking pulse. Here, a locking frequency $\omega_{1} / 2 \pi$ of $20 \mathrm{kHz}$ was applied with pulse powers ranging from 5.1 to $18.5 \mathrm{~W}$. In analogy to the experiments in the laboratory frame, the spin-lock rates $\left(1 / T_{1 \rho}\right)$ were extracted from variable-temperature magnetization transients $M_{\rho}\left(t_{\text {lock }}\right)$. Again, the corresponding transients were parameterized with stretched exponentials determined by $1 / T_{1 \rho}$ and the stretching exponent $\gamma_{1}$. The temperature dependence of the diffusion-induced rates $1 / T_{1(\rho)}$ was 
analyzed in the frame of Arrhenius plots to deduce activation energies and to estimate $\mathrm{Li}^{+}$ motional correlation rates.

\section{Results}

\subsection{Structural characterization}

\subsubsection{Polyanionic substructure}

Solid solutions within $\mathrm{Li}_{4} \mathrm{Ge}_{1-x} \mathrm{Sn}_{x} \mathrm{~S}_{4-y} \mathrm{Se}_{y}$ were synthesized in order to study the correlation between composition, structural changes and ionic mobility in the thio-LISICON family. Highresolution neutron powder diffraction (see Figure S1) was performed for all compositions allowing a characterization of the anionic framework as well as the lithium substructure. An exemplary Rietveld refinement, relative to $\mathrm{Li}_{4} \mathrm{GeSe}_{4}$, is reported in the Supporting Information, Figure S2, showing the good agreement between the calculated and the experimental diffraction profiles. In addition, the crystallographic information format (CIF) files obtained from Rietveld refinements and all the obtained diffractograms can be found in the Supporting Information. All compounds along the solid solution series are well described by the orthorhombic Pnma space group (No. 62), and only minor impurity phases $(<7 w t . \%)$ can be observed for few compositions (Table S2).

Unit cell expansion. The evolution of the unit cell size against composition, reported in Figure 2 , shows a linear increase of the lattice volume upon substitution of $\mathrm{Ge}\left(r_{\mathrm{Ge}}=0.39 \AA\right)$ by $\mathrm{Sn}\left(r_{\mathrm{Sn}}\right.$ $=0.55 \AA)$ and $\mathrm{S}\left(r_{\mathrm{S}}=1.84 \AA\right)$ by $\mathrm{Se}\left(r_{\mathrm{Se}}=1.98 \AA\right)$ driven by the larger ionic radii. ${ }^{57}$ Such linear increase of the lattice volume obeys Vegard's law, therefore confirming the successful synthesis of stable solid solutions. Moreover, the good agreement between the $\mathrm{Ge} / \mathrm{Sn}$ and $\mathrm{S} / \mathrm{Se}$ occupancies obtained from Rietveld refinements with the nominal values further corroborates the full incorporation of the substituents in the solid solutions (see Figure S3). It should be noted, however, that the trend of the lattice parameters against compositions does not always reflect the monotonic trend of the unit cell volume. These deviations from linearity are common in this class of materials that show a tendency of modifying the lattice parameters upon structural rearrangement (vide infra)..$^{34,39,42}$ The lattice parameters of all solid solutions are reported in the Supporting Information, Figure S4 - S7.

Upon closer inspection of the trend of the lattice volume against composition shown in Figure

2 , it can be noticed that the relative unit cell expansion stemming from anionic substitutions ( $16 \%)$ is more pronounced compared to the cationic $(\sim 5 \%)$. This behavior may be caused by the larger number of anions that can be substituted compared to the single central cation. 
Normalizing the unit cell volume expansion by the substitution degree (i.e. maximum number of possible substituents: 4 and 1 for anionic and cationic substitution, respectively), cationic substitutions generate a slightly more pronounced expansion $(\sim 5 \%)$ compared to the anionic $(\sim 4 \%)$. In order to further investigate the underlying reasons behind this behavior, the local effect of the substituents on the $M C h_{4}$ and lithium polyhedral volumes needs to be examined.
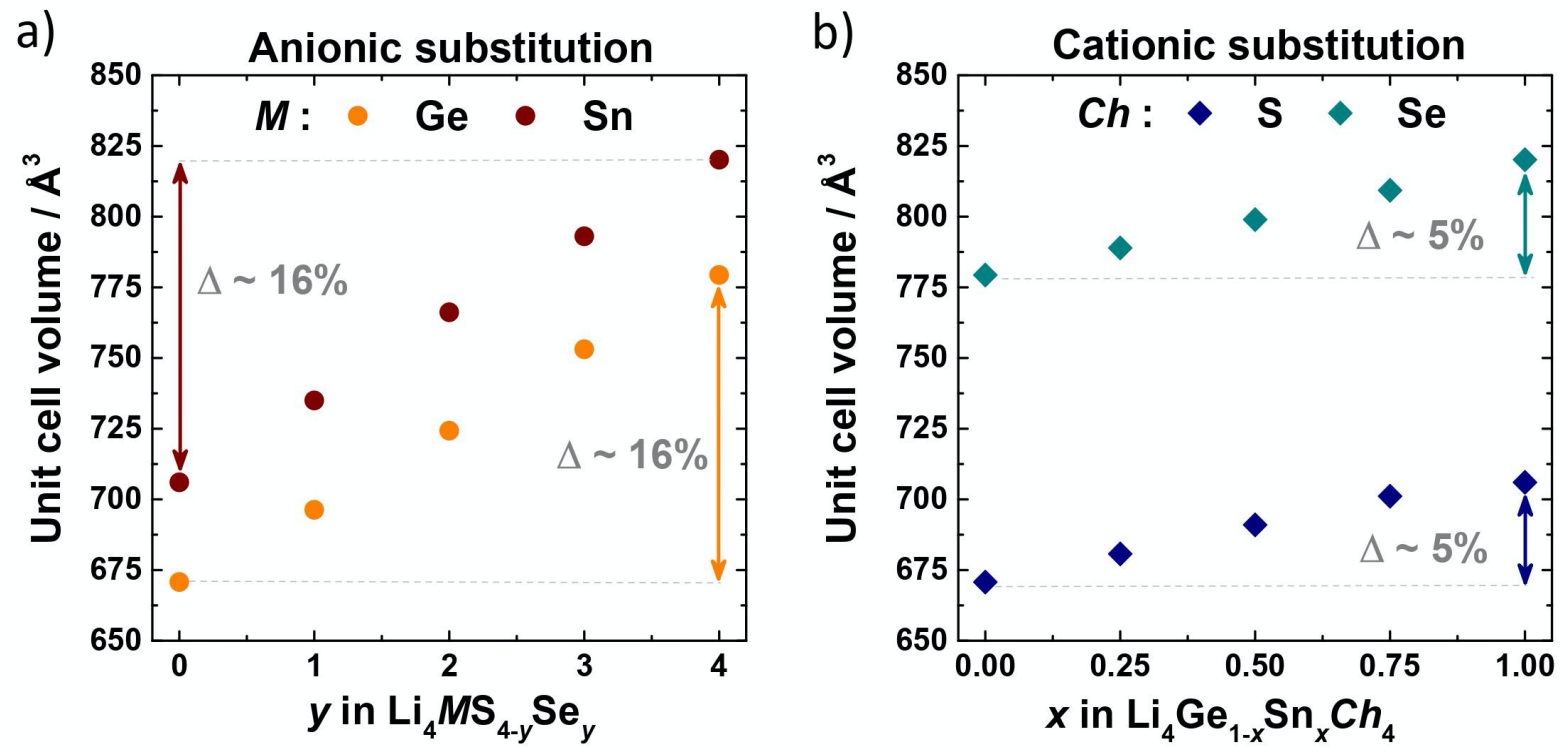

Figure 2: Increasing lattice volume stemming from the incorporation of (a) larger Se anions for $S$ and (b) Sn for Ge cations. The unit cell expansion follows Vegard's law, confirming the successful synthesis of stable solid solutions. Considering the full substitution range, the anionic substitutions generate a more pronounced lattice expansion compared to the cationic.

$M C h_{4}$ expansion. The influence of the cationic and anionic substitutions on the $M C h_{4}$ size is shown in Figure $3 \mathrm{a}$ and $\mathrm{b}$ in which the experimental volume of the $M C h_{4}$ units is reported as function of composition of the solid solutions. The cationic substitutions expand the $M C h_{4}$ volume more $(\sim 24 \%)$ when compared to the anionic $(\sim 20 \%)$ substitutions.

As the larger $M C h_{4}$ volume expansion with cationic substitution may be caused by the slightly larger difference of the ionic radii of the cations compared to the anions (i.e. $\Delta_{\mathrm{Sn}-\mathrm{Ge}}^{r}=0.16 \AA$ and $\Delta_{\mathrm{Se}-\mathrm{S}}^{r}=0.14 \AA$ ), the influence of the ionic radii of the substituents on the $M C h_{4}$ volume needs to be examined. Figure $3 c$ shows the experimental average $M-C h$ distance $\left(d_{M-C h}^{E}\right)$ as function of a calculated theoretical average distance $\left(d_{M-C h}^{C}\right)$ which is based purely on the ionic radii of the constituents of the tetrahedral unit:

$$
d_{M-C h}^{C}=\left(r_{G e} n_{G e}+r_{S n} n_{S n}\right)_{M}+\left(\frac{r_{S} n_{S}+r_{S e} n_{S e}}{4}\right)_{C h}
$$


Where, $r$ indicates the Shannon ionic radius ${ }^{57}$ of a specific element and $n$ is the number of times that element is present in the $M C h_{4}$ unit. The trend in Figure $3 \mathrm{c}$ shows a linear relation between experimental and theoretical values, indicating that the $M-C h$ bond length is mainly driven by the different ionic radii of the elements, which is in good agreement with the hard spheres model of the bond lengths.

a)

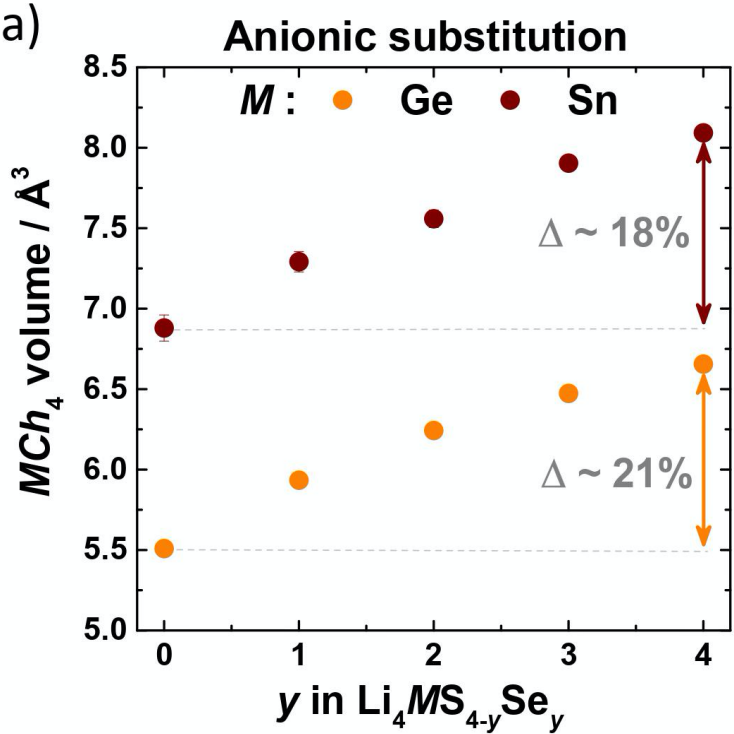

c)

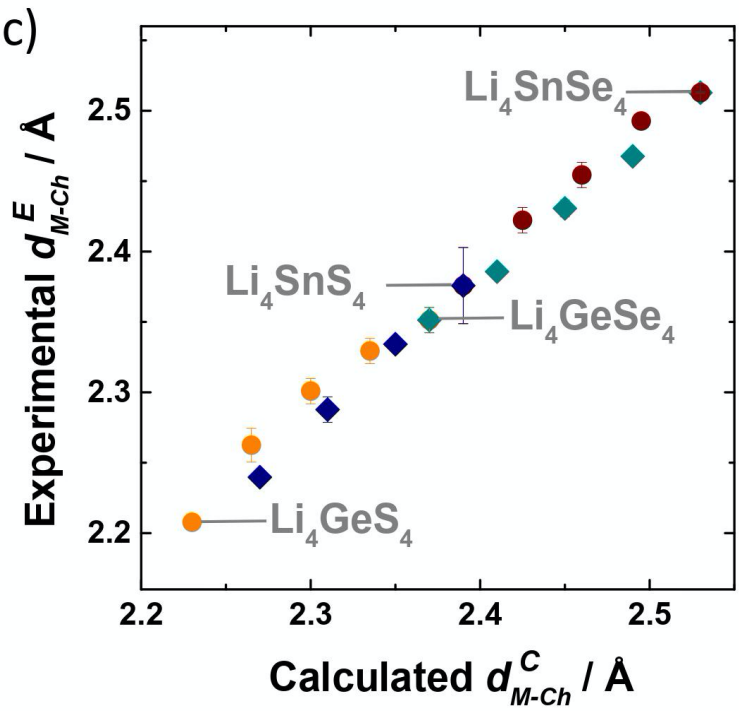

b)
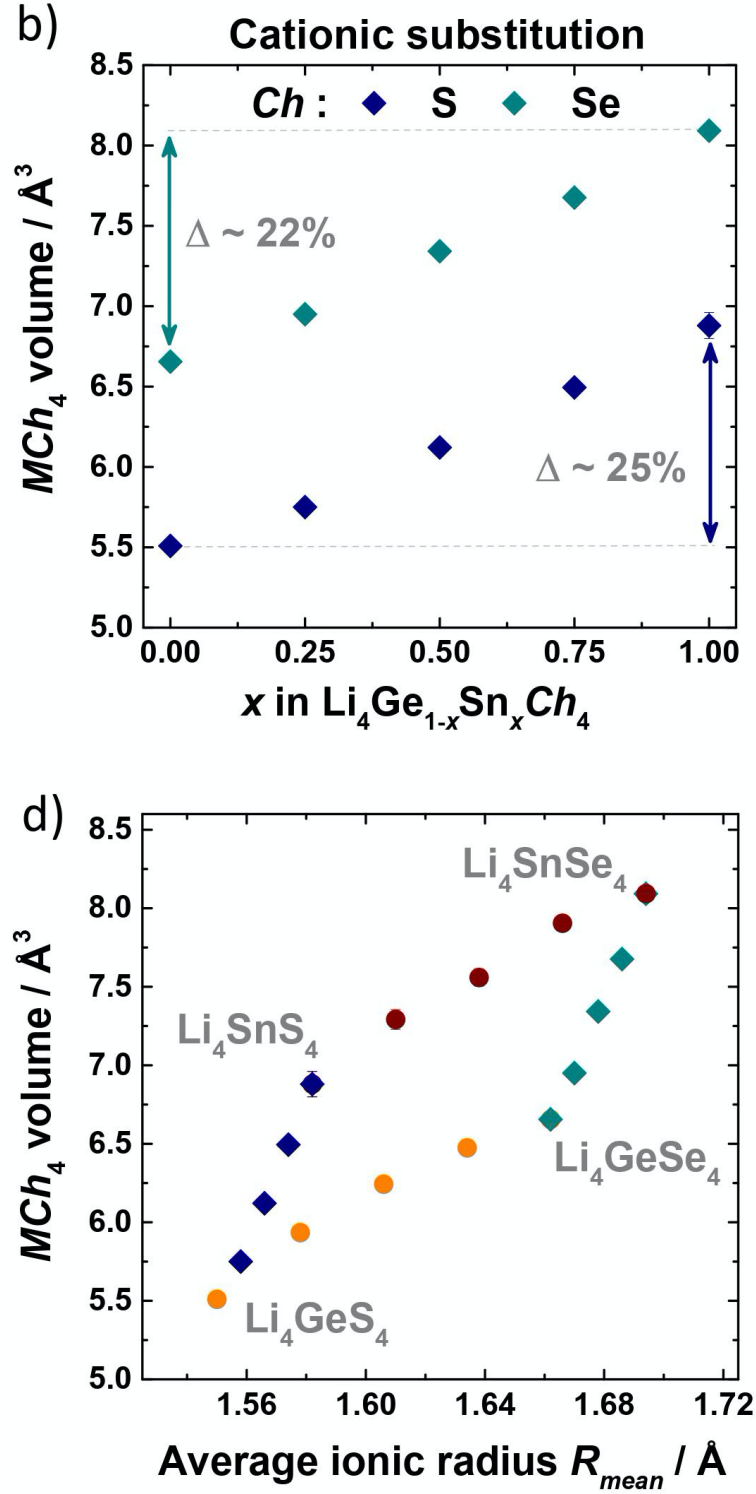

Figure 3: $(a, b)$ Modifications of the experimental $M C h_{4}$ volumes as function of the composition of the solid solutions. (c) The experimental M-Ch bond distance reported as function of the theoretical values reveals that the size of the $M C h_{4}$ units is mainly driven by the radii of elements composing the tetrahedral units. (d) The experimental $\mathrm{MCh}_{4}$ volume reported as a function of the average ionic radius of the elements composing the tetrahedral unit shows that 
cationic substitutions have a greater impact on the $\mathrm{MCh}_{4}$ size compared to the anionic substitutions.

As the expression of $d_{M-C h}$ only considers the average bond length between the central cation and the anion, to investigate the role of the different substituents on the size of the whole $M C h_{4}$ units, in Figure $3 \mathrm{~d}$ the volume of the $M C h_{4}$ units is reported as function of the average ionic radius of the five elements that compose the tetrahedra, expressed as:

$$
R_{\text {mean }}=\left(\frac{r_{S} n_{S}+r_{S e} n_{S e}+r_{G e} n_{G e}+r_{S n} n_{S n}}{5}\right) .
$$

The trends described in Figure $3 \mathrm{~d}$ differ from Figure $3 \mathrm{c}$ as $R_{\text {mean }}$ describes the size of the whole $M C h_{4}$ units, while $d_{M-C h}$ only considers one single $M-C h$ bond length. As can be observed in Figure 3d, anionic and cationic substitutions describe linear trends; albeit, with different slopes. In agreement with the trend reported in Figure $3 \mathrm{a}$ and $\mathrm{b}$, the higher relative slope of the $\mathrm{Li}_{4} \mathrm{Ge}_{1 \text {. }}$ ${ }_{x} \mathrm{Sn}_{x} \mathrm{Ch}_{4}$ solid solutions indicates that the $\mathrm{MCh}_{4}$ volume expansion is stronger when cationic substitutions are performed.

Considering the whole substitution range, i.e. 4 anions and 1 cation, the faster expansion of the $M C h_{4}$ units upon cationic substitution does not reflect the unit cell volume evolution (Figure 2), which is stronger for anionic substitutions. In other words, the larger expansion of the unit cell when anionic substitutions are performed (up to four anions) cannot be explained based solely on the size difference of the $M C h_{4}$ units, since these are expanded more by cationic substitution, therefore hinting to important contributions of other factors on the unit cell expansion.

Lithium polyhedral volumes. To further investigate the influence of the substituents on the structure of these materials, the lithium polyhedral volumes, calculated as an average of tetrahedral and octahedral volumes weighted by the relative lithium occupancies, are reported as function of the composition of the solid solutions in Figure 4. While this averaging does not provide a view on the different polyhedra themselves (see $\mathrm{Li}^{+}$substructure below), it provides insight on the average size of the $\mathrm{Li}^{+}$coordination environments that affect the unit cell volume. The lithium polyhedral expansion is affected in a different fashion by anionic and cation substitution. The anionic substitution has a strong impact on the lithium polyhedral size, while the cationic substitutions have only a marginal effect. Within the whole substitution range, the relative expansion of the $\mathrm{LiCh}_{x}$ volume caused by the different substituents is very similar to the unit cell expansion: anionic substitutions expand both unit cell and $\mathrm{LiCh}_{x}$ volume by $\sim 15 \%$ 
while cationic substitutions have only minor effects on the volume expansion (i.e. $\sim 5 \%)$. This behavior is a strong evidence of the interplay among the unit cell volume and the $\mathrm{Li}^{+}$ environment.
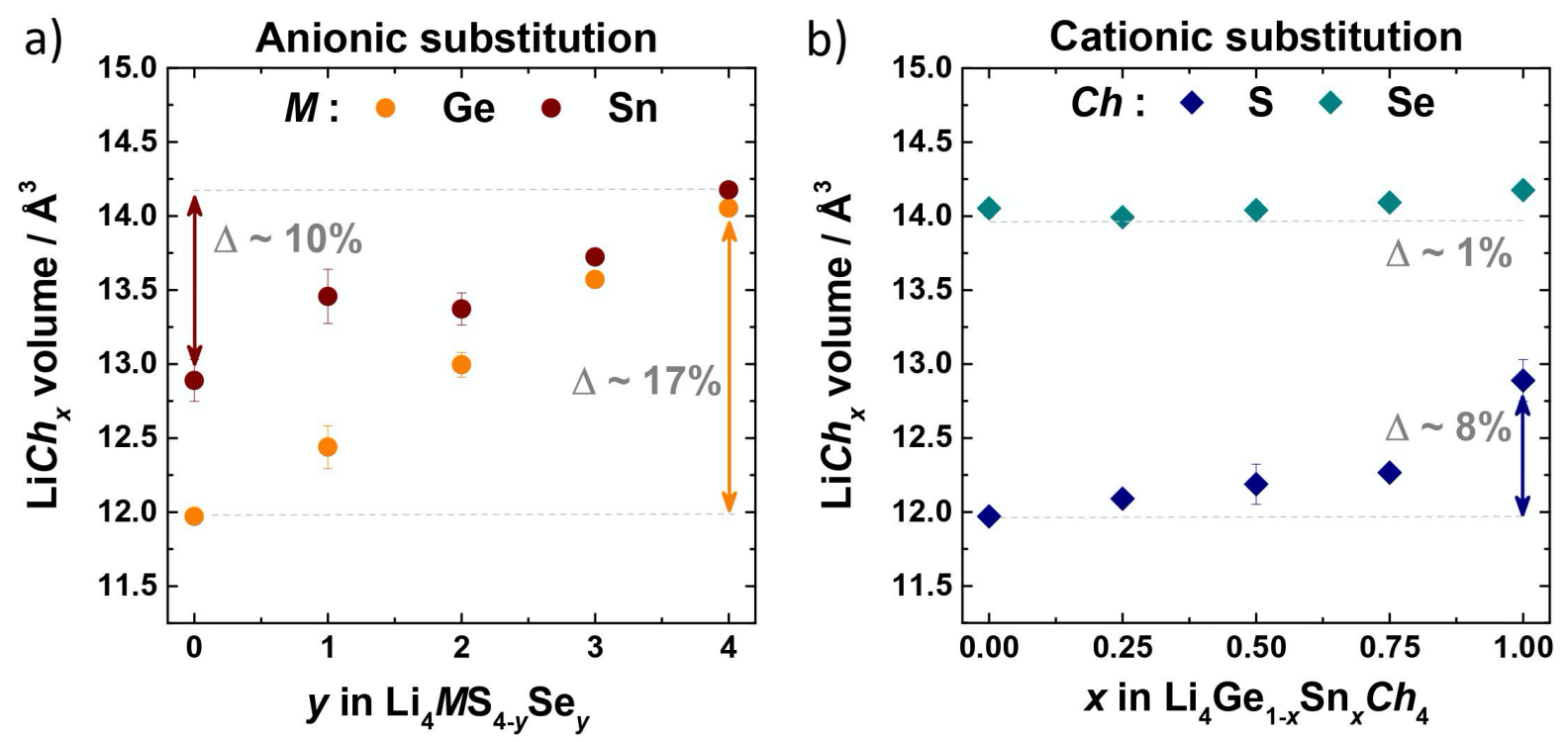

Figure 4: Occupancy weighted average lithium polyhedral volumes as function of composition of the solid solutions. (a) The anionic substitutions generate a pronounced expansion of the lithium polyhedral volumes, while (b) the cationic substitutions have only a minor effect on their size.

Upon closer inspection of Figure 4, it can be noticed that the compositions along the $\mathrm{Li}_{4} \mathrm{Ge}_{1 \text { - }}$ ${ }_{x} \mathrm{Sn}_{x} \mathrm{~S}_{4}$ cationic linear substitution with $x=1$, and the $\mathrm{Li}_{4} \mathrm{SnS}_{1-y} \mathrm{Se}_{y}$ anionic linear substitution with $y=0$ and 1 result to be out of trend compared to the other samples along the solid solution. The reason for this deviation is likely due to the lithium occupancy on the $\operatorname{Li}(4)$ position that, while residing in a octahedral pocket, presents a coordination number closer to five (vide infra). However, an octahedral coordination volume for $\mathrm{Li}(4)$ has been considered for sake of simplicity and consistency with other compositions, therefore resulting in slightly larger average lithium polyhedral volumes for $\mathrm{Li}_{4} \mathrm{SnS}_{4}$ and $\mathrm{Li}_{4} \mathrm{SnS}_{3} \mathrm{Se}$.

\subsubsection{Lithium substructure}

Besides the study of the structural changes of the anionic sublattice, neutron diffraction allows precise analyses of the lithium positions and occupancies in the unit cell. Thus, in order to probe the differences in the lithium substructure brought up by the elemental substitutions, the capacity of all possible sites in the cell to host lithium was investigated. Starting from the 
structural models proposed previously, ${ }^{34,35,38,53}$ lithium occupancy was refined for all possible available sites in the unit cell. The atomic coordinates reported in Table S1 were used as starting point, and the resultant occupancy on the various $\mathrm{Li}^{+}$positions is reported as function of composition in Figure 5a. Further information about the refinement protocol and the utilized constraints can be found in the Supplementary Discussion 1. While all the studied materials crystallize in the same Pnma space group and possess identical anionic frameworks, the lithium substructure drastically changes based on the compositions and on the lattice volumes. For all solid solution series, $75 \%$ of the total lithium occupies tetrahedral positions and the remaining $25 \%$ resides in octahedral interstices (Figure 5a). The ratio of lithium occupancies on tetrahedral and octahedral positions remains unchanged along the substitution series. Below we will introduce the different $\mathrm{Li}^{+}$substructures found in these solid solutions.

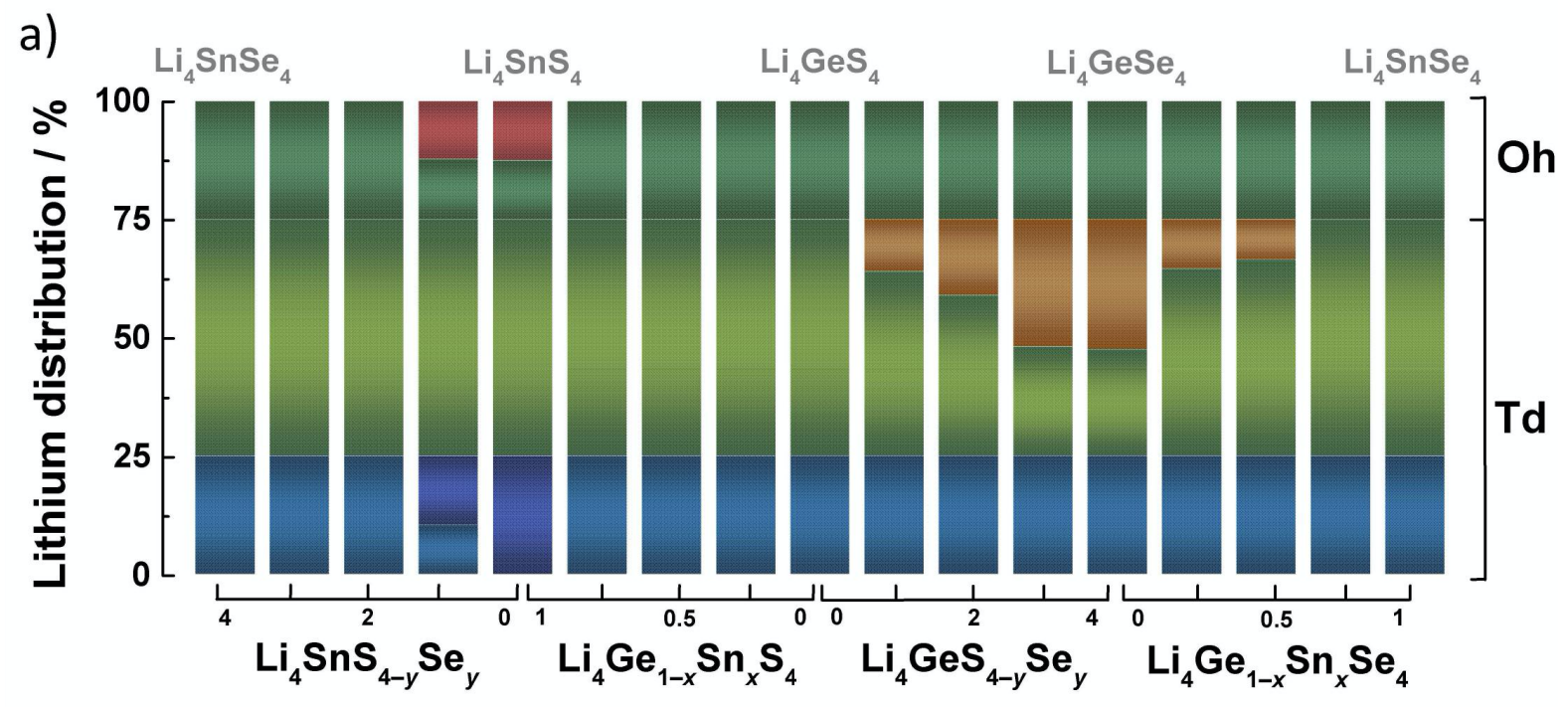

b)

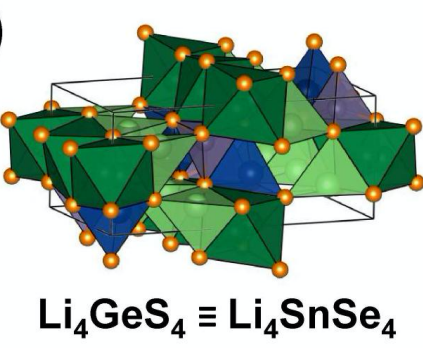

Ch $(8 d, 4 c)$

- $M(4 c)$ c)

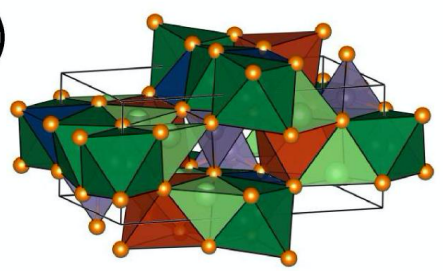

$\mathrm{Li}_{4} \mathrm{SnS}_{4}$ d)

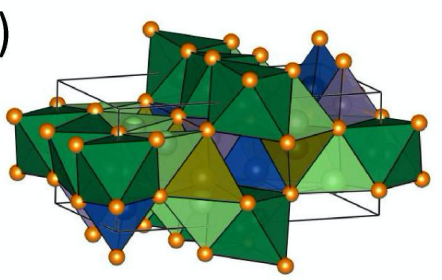

$\mathrm{Li}_{4} \mathrm{GeSe}_{4}$
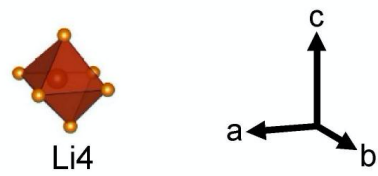

$\begin{array}{lllll}\mathrm{Li} 1 & \mathrm{Li} 1 & \mathrm{Li} 2 & \mathrm{Li}{ }^{\prime} & \mathrm{Li} 3\end{array}$

$\begin{array}{llll}(4 c) & (4 c) & (8 d) & (8 d) \quad(8 d)\end{array}$

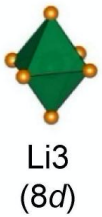

$(4 c)$

Figure 5: a) Lithium distribution on the different sites as function of the composition. Along the solid solution series $75 \%$ of the total lithium occupies tetrahedral positions while the remaining $25 \%$ resides in octahedral interstices. Polyhedral representation of the (b) $\mathrm{Li}_{4} \mathrm{GeS}_{4}$ and $\mathrm{Li}_{4} \mathrm{SnSe}_{4}$ 
together with (c) $\mathrm{Li}_{4} \mathrm{SnS}_{4}$ and (d) $\mathrm{Li}_{4} \mathrm{GeSe}_{4}$ unit cells. While $\mathrm{Li}_{4} \mathrm{GeS}_{4}$ and $\mathrm{Li}_{4} \mathrm{SnSe} \mathrm{H}_{4}$ possess an ordered lithium substructure, $\mathrm{Li}_{4} \mathrm{SnS}_{4}$ and $\mathrm{Li}_{4} \mathrm{GeSe}_{4}$ show some degree of disorder.

i) $\mathrm{Li}_{4} \mathrm{GeS}$. With regard to the lithium sublattice of $\mathrm{Li}_{4} \mathrm{GeS}_{4}, \mathrm{Li}^{+}$ions occupy three inequivalent crystallographic positions: $\operatorname{Li}(1)$ and $\operatorname{Li}(2)$ sites resides in tetrahedral voids (Wyckoff $4 c$ and $8 d$, respectively) and $\mathrm{Li}\left(3\right.$ ) in octahedral interstices (Wyckoff $8 d$ ). The unit cell of $\mathrm{Li}_{4} \mathrm{GeS}_{4}$ and the relative lithium occupancies are reported in Figure 5. The $\operatorname{Li}(3)$ sites are arranged in an edge-sharing chain along the $b$-axis representing the main pathway for ionic diffusion. ${ }^{36,37}$ The $\mathrm{Li}(1)$ tetrahedra share edges with two adjacent $\mathrm{Li}(3)$ octahedra bridging the ionic jumps within the linear chain along the $b$-direction. ${ }^{34}$ Moreover, each Li(3) octahedra shares faces with two $\mathrm{Li}(2)$ sites forming $\mathrm{Li}(2)-\mathrm{Li}(3)-\mathrm{Li}(2)$ units in the $a c$-plane that support fast ionic jumps. ${ }^{34,36,37}$ While fast ionic jumps are allowed within these linear units, the long-range ionic transport occurs along the $b$-direction via the octahedral $\operatorname{Li}(3)$ units bridged by the $\operatorname{Li}(1)$ sites. ${ }^{34}$ These chains are isolated and allow diffusion only in one direction. All these characteristic are highlighted in Figure 6a that shows the linear octahedral chain along the $b$-direction and the local $\mathrm{Li}(2)-\mathrm{Li}(3)-\mathrm{Li}(2)$ units in the ac-plane.

ii) $\mathrm{Li}_{4} \mathrm{SnS} \mathrm{S}_{4}$. Upon step-wise substitutions of $\mathrm{Sn}$ for $\mathrm{Ge}_{\text {in }} \mathrm{Li}_{4} \mathrm{Ge}_{1-x} \mathrm{Sn}_{x} \mathrm{~S}_{4}$, the lithium substructure retains the same character of $\mathrm{Li}_{4} \mathrm{GeS}_{4}$ for $x \leq 0.75$, while it drastically changes for $\mathrm{Li}_{4} \mathrm{SnS}_{4}{ }^{34}$ The unit cell of $\mathrm{Li}_{4} \mathrm{SnS}_{4}$ and the relative lithium occupancies are reported in Figure 5. In this structure, the lithium ions occupy four inequivalent crystallographic positions: $\operatorname{Li}(3)$ and $\operatorname{Li}(2)$, already found in $\mathrm{Li}_{4} \mathrm{GeS}_{4}$, a tetrahedral $\mathrm{Li}\left(1^{\prime}\right.$ ) position (Wyckoff $4 c$ ) and an additional octahedral $\operatorname{Li}(4)$ site (Wyckoff $4 c$ ). Here, we use the terminology of $\operatorname{Li}(1$ ') to denote that $\operatorname{Li}(1)$ repositions itself along the $c$-direction, which can be viewed as a flip of the tetrahedron it belongs to. Similar to $\mathrm{Li}_{4} \mathrm{GeS}_{4}$, the $\mathrm{Li}(3)$ octahedra form linear edge-sharing chains along the $b$-direction that support ionic diffusion. The ionic jumps between the octahedral $\mathrm{Li}(3)$ sites are now bridged by the $\mathrm{Li}\left(1^{\prime}\right)$ positions. $\mathrm{In}_{\mathrm{Li}_{4}} \mathrm{SnS}_{4}, \mathrm{Li}\left(1^{\prime}\right)$ share faces with two adjacent $\mathrm{Li}(3)$ octahedra, while in $\mathrm{Li}_{4} \mathrm{GeS}_{4} \mathrm{Li}(1)$ only share edges with $\mathrm{Li}(3)$ (Figure 6a, b). ${ }^{35,38}$ Therefore, the repositioning of $\operatorname{Li}(1)$ into $\operatorname{Li}\left(1^{\prime}\right)$ for the thiostannate, improves the connectivity of the polyhedra within the linear chains, enhancing the ionic mobility, as recently shown. ${ }^{34}$ Moreover, in $\mathrm{Li}_{4} \mathrm{SnS}_{4}$, lithium ions occupy another octahedral $\mathrm{Li}(4)$ position resulting in a reduced occupancy of $\operatorname{Li}(3)$ (Figure 5). ${ }^{35,38}$ The $\mathrm{Li}(4)$ site plays a crucial role in the ionic diffusion as it bridges the one-dimensional chains formed by $\mathrm{Li}(3)$ and the surrounding $\mathrm{LiS}_{4}$ tetrahedra (i.e. 
$\operatorname{Li}\left(1^{\prime}\right)$ and $\left.\operatorname{Li}(2)\right)$, thereby activating a three-dimensional conduction behavior via $\operatorname{Li}(2)-\operatorname{Li}(4)$ and $\operatorname{Li}\left(1^{\prime}\right)-\operatorname{Li}(4)$ exchange..$^{34}$

Considering the effect of $\operatorname{Li}\left(1^{\prime}\right)$ and $\operatorname{Li}(4)$ on the lithium polyhedral connectivity shown in Figure $6 b$, it is evident that the lithium redistribution in $\mathrm{Li}_{4} \mathrm{SnS}_{4}$ allows a greater $\mathrm{Li}-\mathrm{Li}$ percolation together with a better polyhedral connectivity, which we have already shown to result in better transport properties compared to the $\mathrm{Li}_{4} \mathrm{GeS}_{4}$ parent phase. ${ }^{34}$

iii) $\mathrm{Li}_{4} \mathrm{SnSe}_{4}$. In line with previous reports, ${ }^{53}$ the lithium substructure of $\mathrm{Li}_{4} \mathrm{SnSe}_{4}$ is identical to $\mathrm{Li}_{4} \mathrm{GeS}_{4}$. Upon anionic substitution in $\mathrm{Li}_{4} \mathrm{SnS}_{4-y} \mathrm{Se}_{y}$, the lithium substructure changes progressively resulting in lithium occupancy for both $\mathrm{Li}(1)$ and $\mathrm{Li}\left(1^{\prime}\right)$ in $\mathrm{Li}_{4} \mathrm{SnS}_{3} \mathrm{Se}$, while it matches the motif of $\mathrm{Li}_{4} \mathrm{SnSe}_{4}$ for $y \geq 2$ (Figure 5 and $6 \mathrm{~d}$ ).

iv) $\mathrm{Li}_{4} \mathrm{GeSe}_{4}$. The lithium substructure of $\mathrm{Li}_{4} \mathrm{GeSe}_{4}$ resembles that of $\mathrm{Li}_{4} \mathrm{GeS}_{4}$, however additional lithium occupancy can be found in a new tetrahedral site (Wyckoff $8 d$ ), which we denote here as $\operatorname{Li}\left(2^{\prime}\right)$. As shown in Figure 5, the lithium occupation on $\operatorname{Li}\left(2^{\prime}\right)$ sites results in reduced occupancy for $\mathrm{Li}(2)$ and represents an inversion of the $\mathrm{Li}(2) \mathrm{S}_{4}$ tetrahedron. The newly found $\mathrm{Li}\left(2^{\prime}\right)$ tetrahedral sites share a face with $\mathrm{Li}(2)$ forming a $\mathrm{Li}(2) / \mathrm{Li}\left(2^{\prime}\right)$ double tetrahedron. This local coordination is shown in Figure 6c. The lithium nuclear density is displaced from the centroid of each single $\mathrm{Li}(2)$ or $\operatorname{Li}\left(2^{\prime}\right)$ tetrahedron and it is relocated towards the center of the shared triangular face. While a trigonal coordination for lithium ions in the typical volumes range of sulfidic materials is unfavorable, the lattice volume expansion caused by the elemental substitutions may make such sites available for lithium occupation. These $\operatorname{Li}(2) / \operatorname{Li}\left(2^{\prime}\right)$ double tetrahedra share edges with other $\operatorname{Li}(2) / \operatorname{Li}\left(2^{\prime}\right)$ double tetrahedra, forming chains along the $c$ axis. Given that, the partially occupied sites define the set of pathways available for possible lithium diffusion, it is likely that the edge-sharing double tetrahedra along the $c$-axis support ionic diffusion. Interestingly, further increase or decrease of the lattice volume results in reduced lithium occupancy on $\mathrm{Li}\left(2^{\prime}\right)$ for $\mathrm{Li}_{4} \mathrm{GeS}_{4-y} \mathrm{Se}_{y}$ and $\mathrm{Li}_{4} \mathrm{Ge}_{1-x} \mathrm{Sn}_{x} \mathrm{Se}_{4}$ (Figure 5a). 


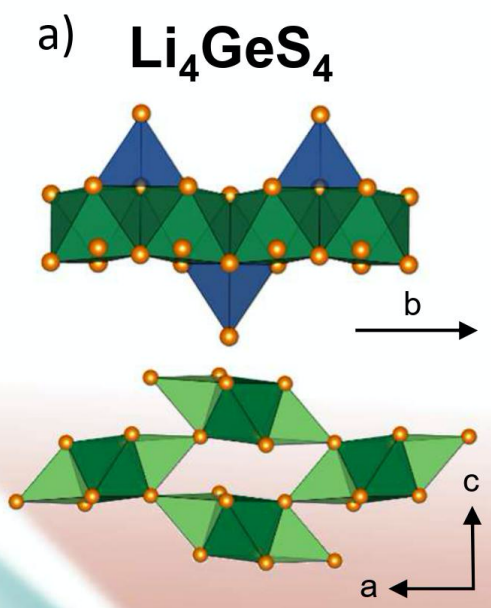

\section{b) $\mathrm{Li}_{4} \mathrm{SnS}_{4}$}

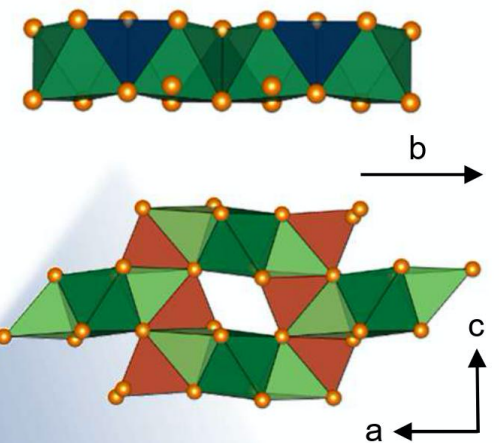

d) $\mathrm{Li}_{4} \mathrm{SnSe}_{4}$

c) $\mathrm{Li}_{4} \mathrm{GeSe}_{4}$
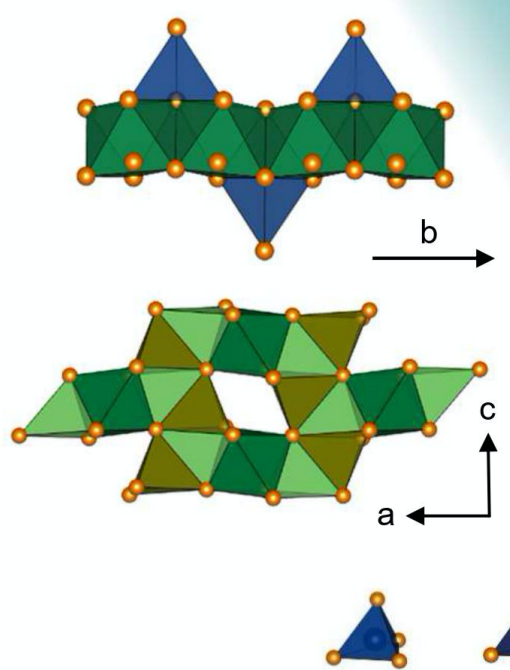

Li1

(4c)

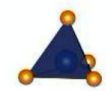

Li1'

(4c)
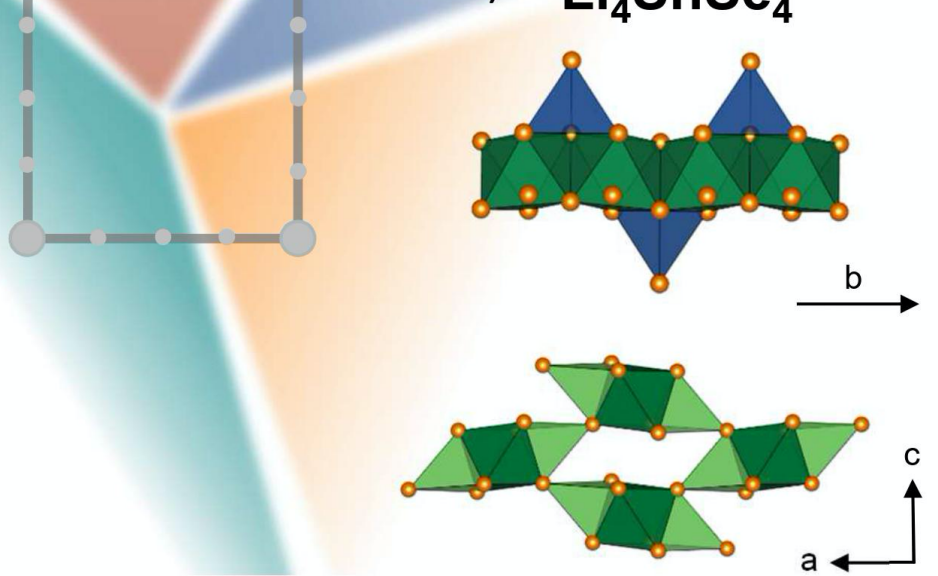

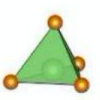

Li2

(8d)

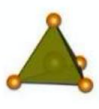

Li2'

$(8 d)$

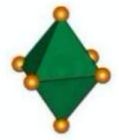

Li3

(8d)

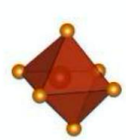

Li4

(4c)

Figure 6: (a-d) Local polyhedral arrangement in the ac-plane and along the b-direction of the different lithium substructures found in the solid solutions. For clarity Li(2) is omitted in the representation along the b-direction, whereas Li(1) is omitted in the ac-plane. Different colors of the background indicate specific regions of the two-dimensional substitution series in which a certain lithium substructure was found.

Upon a closer inspection of the lithium distribution against composition reported in Figure 5a, it can be noticed that, lithium disorder that is usually highly beneficial for the ionic transport, is affected by different anion/cation combinations rather than the presence of particular chemical elements in the structure. ${ }^{58}$ The origin of this behavior can likely be traced back to the different size of the $\mathrm{MCh}_{4}$ or $\mathrm{LiCh}_{x}$ units, that may generate optimal volumes and charge disorder favorable for disordered lithium distribution..$^{26,58}$ 


\subsection{Ionic transport}

Impedance spectroscopy. The ionic transport properties of the $\mathrm{Li}_{4} \mathrm{Ge}_{1-x} \mathrm{Sn}_{x} \mathrm{~S}_{4-y} \mathrm{Se}_{y}$ series were analyzed by temperature-dependent impedance spectroscopy. The impedance responses within the explored temperature range are similar throughout the solid solution series and consist of a well-resolved semicircle followed by a spike in the lower frequency region (Figure 7a). The obtained data were fitted with an equivalent circuit consisting of one parallel constant phase element (CPE)/resistor in series with a CPE (insert in Figure 7a). The capacitance of the CPE used to fit the semicircle is in the range of $10^{-11} \mathrm{~F} / \mathrm{cm}^{2}$, and all impedance spectra exhibit $\alpha-$ values of $\sim 0.9$, all of which indicate a predominant bulk contribution to the impedance response. ${ }^{59}$ All samples in the $\mathrm{Li}_{4} \mathrm{Ge}_{1-x} \mathrm{Sn}_{x} \mathrm{~S}_{4-y} \mathrm{Se}_{y}$ series show Arrhenius behavior within the temperature range of 283-333 K (Figure 7b). The room-temperature ionic conductivity values for the solid solution series and the activation energy for ionic diffusion, as determined from a linear fit of the Arrhenius plots, are reported in Figure 7c and d, respectively. While consistent with our previous studies, ${ }^{34}$ the herein observed ionic conductivity for $\mathrm{Li}_{4} \mathrm{SnS}_{4}$ and $\mathrm{Li}_{4} \mathrm{SnSe}_{4}$ are significantly lower compared with the values reported by Kaib et al. ${ }^{35,53}$ likely stemming from the differences in the synthesis protocols and parameters employed for the electrical characterization. ${ }^{60}$ 

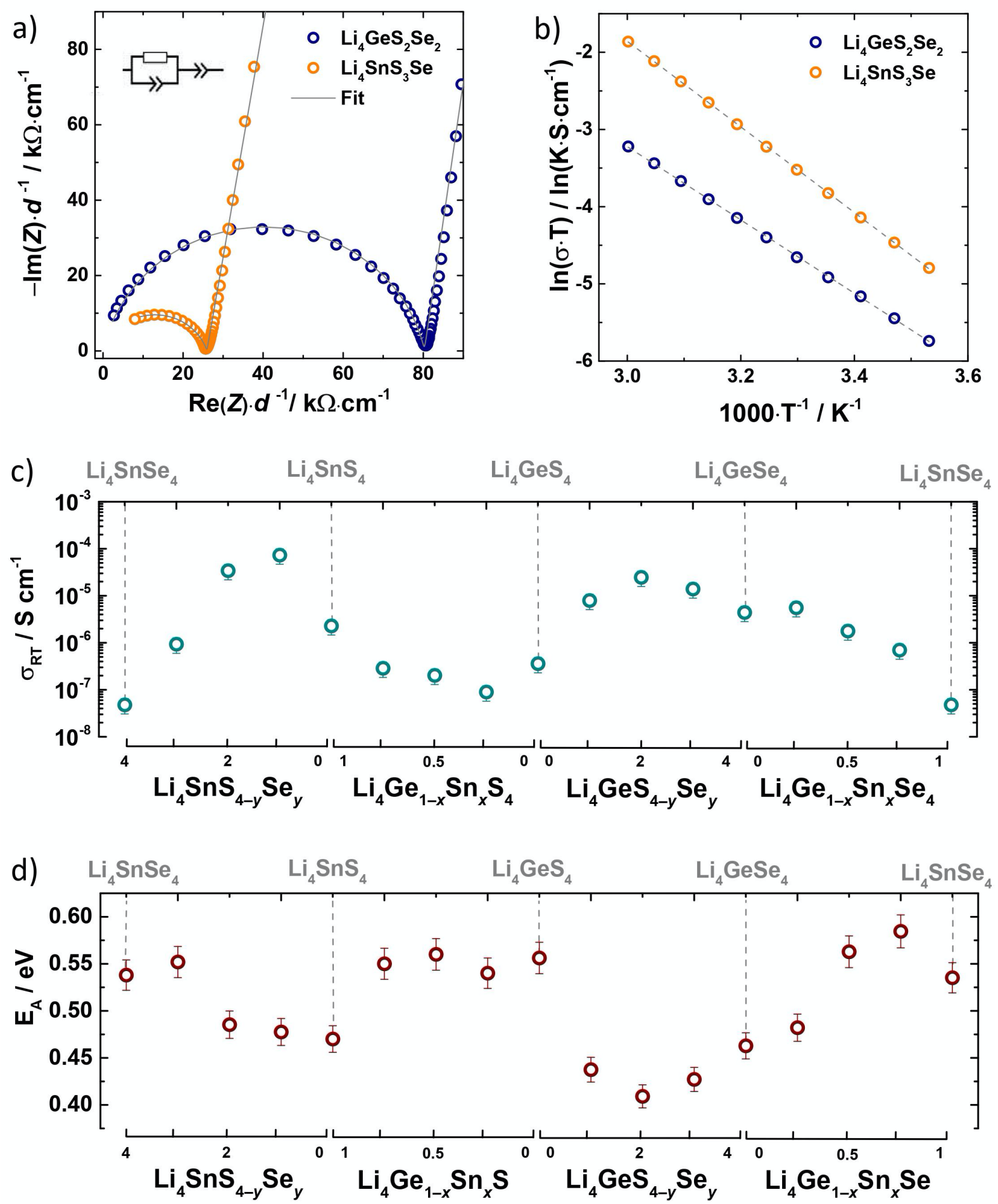

Figure 7: (a) Exemplary Nyquist plots at room-temperature, showing the impedance response (open circles) and the fit (gray line) with the employed equivalent circuit (insert). (b) Exemplary Arrhenius plots of the conductivity values obtained from temperature-dependent impedance spectroscopy and the respective linear fit (gray dotted line). (c) Room-temperature ionic conductivity, $\sigma_{R T}$, and (d) activation energy, $E_{A}$, as a function of composition in $\mathrm{Li}_{4} \mathrm{Ge}_{1-x} \mathrm{Sn}_{x} \mathrm{~S}_{4-}$ ${ }_{y} S e_{y}$. Uncertainties of the room-temperature ionic conductivity and the activation energies 
values were obtained based on the measurement of three separately synthesized samples per composition from our previous study. ${ }^{34}$

The room temperature ionic conductivity and the activation energy are significantly affected by the isovalent substitutions in $\mathrm{Li}_{4} \mathrm{Ge}_{1-x} \mathrm{Sn}_{x} \mathrm{~S}_{4-y} \mathrm{Se}_{y}$. These drastic changes indicate that the transport properties of this class of materials can be tailored by the modification of the lithium substructure and anionic framework without any change of the carrier concentration. However, a clear explanation of these trends is not possible only considering the different compositions of the solid solutions, therefore, other descriptors must be employed to better comprehend the evolution of the transport properties of these materials (vide infra).

Nuclear Magnetic Resonance. ${ }^{7} \mathrm{Li}$ NMR spin-lattice relaxation experiments were performed in both the laboratory and the rotating frame of reference to obtain information on the $\mathrm{Li}^{+}$jump rates and activation energies of the underlying motional processes of the two-dimensional substitution series end-members, namely $\mathrm{Li}_{4} \mathrm{GeS}_{4}, \mathrm{Li}_{4} \mathrm{SnS}_{4}, \mathrm{Li}_{4} \mathrm{GeSe}_{4}$ and $\mathrm{Li}_{4} \mathrm{SnSe}_{4}$. These compositions were selected for the NMR experiments due to their different lithium substructure and unit cell volume, therefore allowing a comprehensive study of the lithium configurations and polyhedral volumes effects on the lithium motion. Moreover, since ${ }^{7} \mathrm{Li}$ NMR spin-lattice relaxation rates measured in the laboratory frame of reference, $1 / T_{1}$, are sensitive to spin fluctuations with rates on the $\mathrm{MHz}$ range $\left(\omega_{0} / 2 \pi=116 \mathrm{MHz}\right)$ and spin-lock NMR rates, $1 / T_{1 \varrho}$, to motional correlation rates in the $\mathrm{kHz}$ range $\left(\omega_{1} / 2 \pi=20 \mathrm{kHz}\right)$, we were able to cover a large dynamic range of hopping processes on different time scales. Here, via variable-temperature $1 / T_{1 \varrho}$ spin-lock ${ }^{7} \mathrm{Li}$ NMR we recorded the complete diffusion-induced rate peak. ${ }^{61}$

The corresponding Arrhenius plots of the spin-lattice relaxation rates, $1 / T_{1}(1 / T)$ and $1 / T_{1 \mathrm{e}}(1 / T)$, for each sample are shown in Figure 8. In the upper graphs, the change of the stretching exponents $\gamma_{(\varrho)}$ are displayed for comparison with the behavior of $1 / T_{1(\varrho)}$. Note that maxima of the relaxation rate are correlated with minima of the stretching exponent. Below, we describe the different lithium dynamics and, adopting the formalism described in the Supplementary Discussion 2, provide an overview on the $\mathrm{Li}^{+}$attempt frequencies and activation energies for ionic motion of the samples examined (Table S3).

i) $\mathrm{Li}_{4} \mathrm{GeS}_{4}$. The ${ }^{7} \mathrm{Li} \mathrm{NMR}$ spin-lattice relaxation rates $1 / T_{1(\mathrm{o})}$ of the thiogermanate are shown in Figure $8 \mathrm{a}$. While the laboratory-frame rate only exhibits a weak temperature dependence with a shallow and broadened peak at $330 \mathrm{~K}$, the spin-lock relaxation rate passes through two rate 
peaks $1 / T_{1 \mathrm{e}}(1 / T)$ appearing at $320 \mathrm{~K}$ and $360 \mathrm{~K}$, respectively. This feature is unique of $\mathrm{Li}_{4} \mathrm{GeS}_{4}$ and it is not observed for any of the other examined compositions (vide infra). The overall response was parameterized with a sum of two Lorentzian shaped relaxation rate peaks according to the model introduced by Bloembergen, Purcell and Pound (BPP $)^{62}$ (solid line). The dashed lines in Figure 8a indicate the individual diffusion-induced rate peaks. The peak located at $T_{\max }=320 \mathrm{~K}$ reveals an activation energy of $0.58 \mathrm{eV}$ and an attempt frequency of $5 \cdot 10^{13} \mathrm{~s}^{-1}$, while the second peak at $T_{\max }=360 \mathrm{~K}$ has to be described with a lower activation energy of $0.53 \mathrm{eV}$ and an attempt frequency of only $4 \cdot 10^{12} \mathrm{~s}^{-1}$. The appearance of the two $1 / T_{10}(1 / T)$ relaxation rate peaks of $\mathrm{Li}_{4} \mathrm{GeS}_{4}$ indicates that $\mathrm{Li}^{+}$diffusivity in this material is stepwise activated and different motion processes dominate at different temperatures.

ii) $\mathrm{Li}_{4} \mathrm{SnS} \mathrm{S}_{4}$. Similar to $\mathrm{Li}_{4} \mathrm{GeS}_{4}$, but more pronounced, in the thiostannate $\mathrm{Li}_{4} \mathrm{SnS}_{4}$ a $1 / T_{1}$ peak appears slightly above $333 \mathrm{~K}$ (Figure 8b). In this temperature region, the magnetization transients show bi-exponential behaviour characterized by two rates $1 / T_{1 \text {,ast }}$ and $1 / T_{1, \text { slow, }}$ pointing to two different spin reservoirs. The rate peak $1 / T_{1, \text { fast }}(1 / T)$ points to a relatively low activation energy of only $0.38 \mathrm{eV}$, which is in line with the measurements reported by Kaib et $a l .{ }^{35}$ This feature, most likely stemming from the disordered lithium substructure, reflects a rapid, localized forward-backward spin fluctuations with characteristic rates on the $\mathrm{MHz}$ time scale.

Contrarily to $\mathrm{Li}_{4} \mathrm{GeS}_{4}$, the spin-lock rate peak of $\mathrm{Li}_{4} \mathrm{SnS}_{4}$ reveals only a single BPP-type peak located at $T_{\max }=333 \mathrm{~K},{ }^{62}$ characterized by an activation energy of $0.54 \mathrm{eV}$ and an attempt frequency of $3 \cdot 10^{13} \mathrm{~s}^{-1}$. Worth noting, the $1 / T_{1, \text { fast }}(1 / T)$ peak does not affect spin-lock relaxation as a corresponding peak $1 / T_{1 \mathrm{l}}$ at sufficiently low temperatures is missing. Obviously, the two methods are sensitive to quite different spin fluctuations in $\mathrm{Li}_{4} \mathrm{SnS}_{4}$. We assume that if several $\mathrm{Li}$ ion jump processes run in parallel not all of them will contribute to the distinct, time-scale dependent NMR responses in equal shares. The process characterized by $0.38 \mathrm{eV}$ is, however, considered as a key feature to explain the enhanced transport properties observed for $\mathrm{Li}_{4} \mathrm{SnS}_{4}$ as it is more pronounced than in the other compounds studied..$^{35}$

iii) $\mathrm{Li}_{4} \mathrm{SnSe}_{4} \cdot{ }^{7} \mathrm{Li} \mathrm{NMR}$ laboratory-frame spin-lattice relaxation in $\mathrm{Li}_{4} \mathrm{SnSe}_{4}$ reveals only a shallow temperature dependence up to $333 \mathrm{~K}$ (Figure 8c); that is controlled by localized or correlated forward-backward $\mathrm{Li}^{+}$jump processes which also influence the $1 / T_{1}$ rates of the other compounds at sufficiently low $T .^{63,64}$ In contrast to the other examined compositions, for $\mathrm{Li}_{4} \mathrm{SnSe}_{4}$ the rate $1 / T_{1}$ does not show any peak-like feature. Spin-lock NMR, however, exhibits 
a rate peak at $T_{\max }=328 \mathrm{~K}$ that, again, can be described with a symmetric Lorentzian-shaped BPP function; ${ }^{62}$ the fit yields a relatively high activation energy of $0.69 \mathrm{eV}$ and an attempt frequency of $7 \cdot 10^{15} \mathrm{~s}^{-1}$.

iv) $\mathrm{Li}_{4} \mathrm{GeSe}_{4}$. The laboratory-frame rate $1 / T_{1}$ of $\mathrm{Li}_{4} \mathrm{GeSe}_{4}$ (Figure $8 \mathrm{~d}$ ) passes through a shallow maximum located at $290 \mathrm{~K}$, likely stemming from the disordered lithium substructure as for $\mathrm{Li}_{4} \mathrm{SnS}_{4}$. Compared to $\mathrm{Li}_{4} \mathrm{SnS}_{4}$ this peak is located at much lower temperatures indicating faster diffusion processes on the angstrom length scale. The spin-lock relaxation rate shows an almost symmetric peak at temperature $T_{\max }=320 \mathrm{~K}$ slightly lower than that of $\mathrm{Li}_{4} \mathrm{SnS} \mathrm{S}_{4}$. The high- $T$ flank of the peak is to be characterized by a relatively high $\mathrm{BPP}^{62}$ activation energy of $0.62 \mathrm{eV}$ and an attempt frequency of $10^{15} \mathrm{~s}^{-1}$. The low- $T$ flank can be characterized by an activation energy very similar to $\mathrm{Li}_{4} \mathrm{GeS}_{4}$, indicating that probably for a limited window at lower temperatures the $\mathrm{Li}^{+}$local motion is similar for these two materials. 

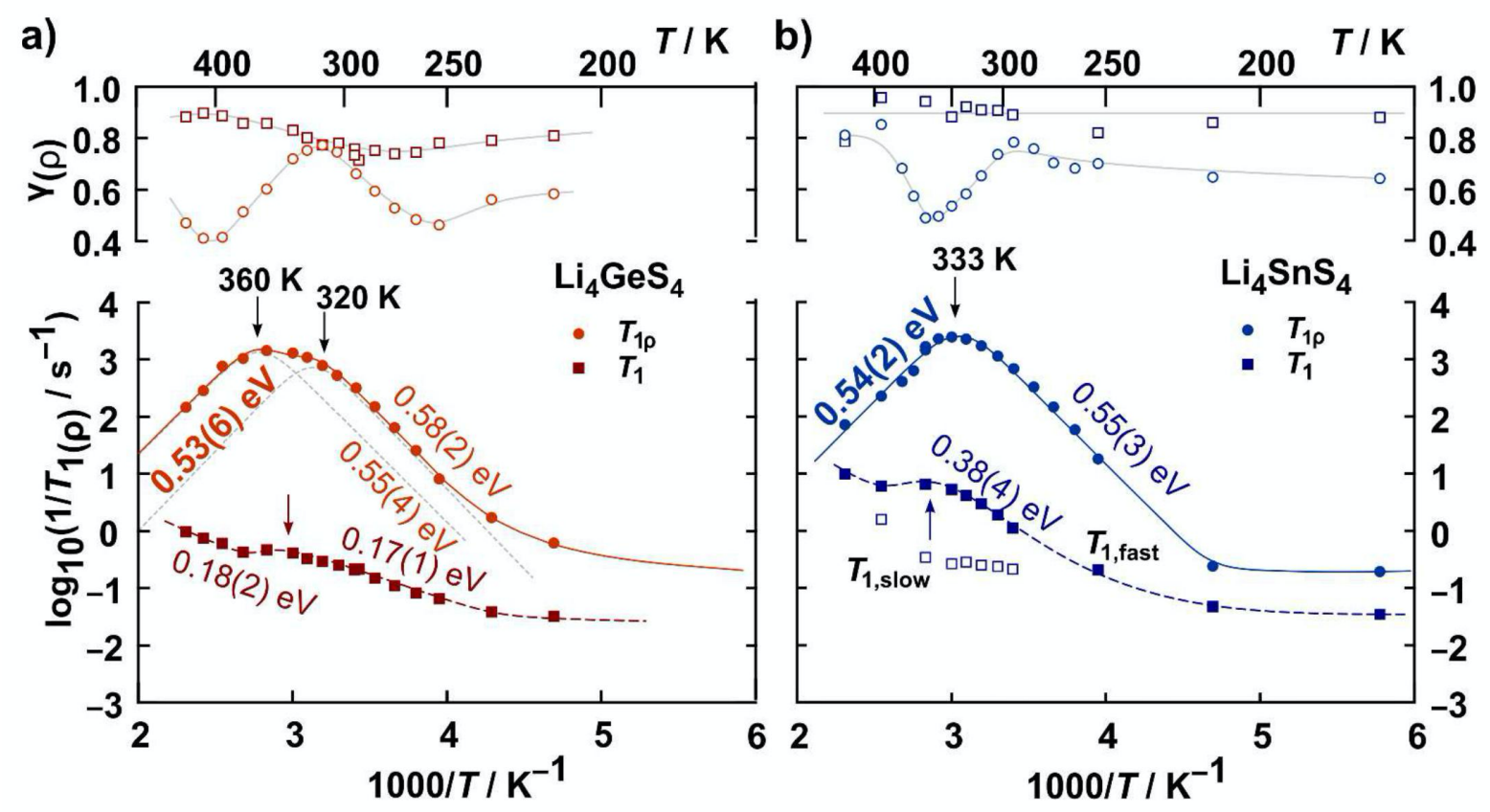

c)

d)
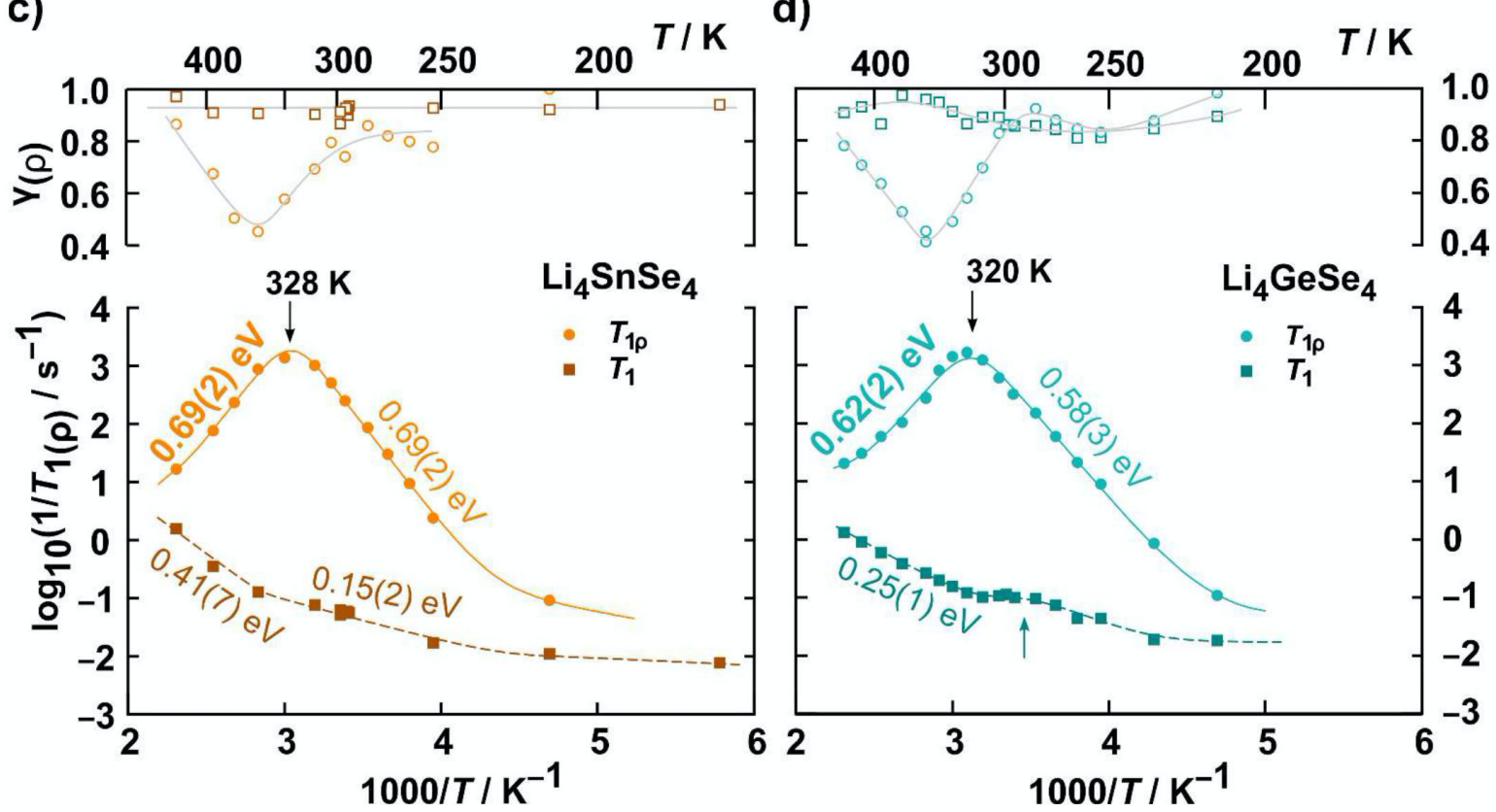

Figure 8: Arrhenius plot of the ${ }^{7} \mathrm{Li}$ NMR spin-lattice relaxation rates in the laboratory frame $1 / T_{1}$ (squares) and rotating-frame 1/T $T_{l \varrho}$ (circles) of (a) $\mathrm{Li}_{4} \mathrm{SnSe}_{4}$, (b) $\mathrm{Li}_{4} \mathrm{SnS}_{4}$, (c) $\mathrm{Li}_{4} \mathrm{GeS}_{4}$ and (d) $\mathrm{Li}_{4} \mathrm{GeSe}_{4}$. Solid lines, parameterizing the behavior of the relaxation rates, represent fits with BPP-type Lorentzian shaped spectral density functions..$^{62}$ Dashed lines are drawn to guide the eye. Activation energies given in bold letters refer to those obtained from BPP fits. They represent the slopes of the respective peaks in the high-T limits. Activation energies of partly linear regions of $1 / T_{1}$ and of the low-T flanks of the $1 / T_{10}$ rate peaks are also included. The temperatures $T_{\max }$ at which the $1 / T_{1 \varrho}$ relaxation rate peaks reach their maximum are reported as well. Upper graphs show the temperature behaviour of the corresponding stretching exponents $\gamma_{(\varrho)}$; lines represent guides to the eye. 


\section{Discussion}

Solid solutions within $\mathrm{Li}_{4} \mathrm{Ge}_{1-x} \mathrm{Sn}_{x} \mathrm{~S}_{4-y} \mathrm{Se}_{y}$ were synthesized in order to study the correlation between composition, structural changes and ionic mobility in the thio-LISICON family. Based on the above seen changes in the structure and transport properties, a number of conclusions can be drawn:

1) Anionic sublattice and lattice expansion. The evolution of the $M C h_{4}$ and lithium polyhedral volume, against composition of the solid solutions shows that the anionic and cationic substitutions have different effects on the polyhedral expansion. The correlation among $\mathrm{MCh}_{4}$ and lithium polyhedral volumes is shown in Figure 9a. While the $M C h_{4}$ is reported on the $x$-axis and the lithium polyhedra on the $y$-axis, this plot is not meant to suggest that the lithium polyhedral volume is a function of the $M C h_{4}$ size, but only to highlight the correlation among these structural factors. The trend in Figure 9a shows that cationic substitutions generate a more pronounced expansion of the $M C h_{4}$ units, while anionic substitutions have a greater impact on the lithium polyhedral size. The larger expansion of the $M C h_{4}$ units upon cationic substitution can be explained considering the slightly larger difference of the ionic radii of the cations compared to the anions (i.e. $\Delta_{\mathrm{Sn}-\mathrm{Ge}}^{r}=0.16 \AA$ and $\Delta_{\mathrm{Se}-\mathrm{S}}^{r}=0.14 \AA$ ). This evidence indicates that the expansion of the $\mathrm{MCh}_{4}$ is mainly driven by the ionic radii of the elements composing such units (Figure 3c). With regard to the lithium polyhedral expansion, it is clear that anionic substitutions have a much greater impact on the volume than the cationic, since the cationic species do not actively take part in the $\mathrm{LiCh}_{\mathrm{x}}$ polyhedra.

The different effects that cationic and anionic substitutions have on the local volumes of the $\mathrm{MCh}_{4}$ and $\mathrm{LiCh}_{\mathrm{x}}$ polyhedra strongly influence the unit cell expansion. Figure $9 \mathrm{~b}$ shows that, while cationic substitutions expand the $M C h_{4}$ units more, the relative unit cell expansion stemming from anionic substitutions is more pronounced compared to the cationic. Therefore, the larger expansion of the unit cell when anionic substitutions are performed cannot be explained based solely on the size difference of the $M C h_{4}$ units. For example, while possessing smaller $M C h_{4}$ volume, the unit cell size of $\mathrm{Li}_{4} \mathrm{GeSe}_{4}$ is substantially larger than $\mathrm{Li}_{4} \mathrm{SnS}_{4}$ (see Figure 9b). Therefore, the size of the $M C h_{4}$ units cannot be the only driving factor of the unit cell expansion. These trends may be caused by the different electric forces among $M \mathrm{~S}_{4}$ or $M \mathrm{Se}_{4}$ units. The widely larger electronic cloud of the Se anions composing the $M C h_{4}$ may generate stronger electrostatic 
repulsion among neighboring units that are, therefore, driven apart resulting in larger unit cell volumes compared with the sulfidic counterpart. ${ }^{65}$

The larger volume expansion of the unit cell upon anionic substitutions correlates well with the lithium polyhedral expansion as shown in Figure 9c. The relative expansion of the $\mathrm{LiCh}_{x}$ volume caused by the different substituents is indeed very similar to the unit cell expansion: within the whole range of substitutions, the anionic substitutions expand both unit cell and $\mathrm{LiCh}_{x}$ volume by $\sim 15 \%$ while cationic substitutions have only minor effects on the volume expansion (i.e. $~ 5 \%$ ).

Therefore, anionic substitutions cause major expansions of the unit cell volume by enlarging the lithium polyhedral volumes, possibly due to repulsion of the anionic "ligands", while cationic substitutions enlarge the $M C h_{4}$ volumes with smaller lattice expansion and barely affecting the lithium polyhedral pathways.

All these correlations highlight the complex interplay of many structural factors that influence the lattice size. In the typically performed one-dimensional substitutions series the lattice expansion would have surely been blamed only to the size of the $M C h_{4}$ tetrahedra, here, by performing a two-dimensional substitution we are able to demonstrate that the $\mathrm{MCh}_{4}$ size is not enough to explain the lattice expansion. The $\mathrm{LiCh}_{x}$ expansion instead seems to play a major role in determining the unit cell volume. This evidence indicates the importance of two-dimensional substitution studies to understand the general role of the substituents on the structural modifications. 
Anionic substitution Cationic substitution $M=\bullet \mathrm{Ge} \bullet \mathrm{Sn} C h=\diamond \mathrm{Se} \bullet \mathrm{S}$
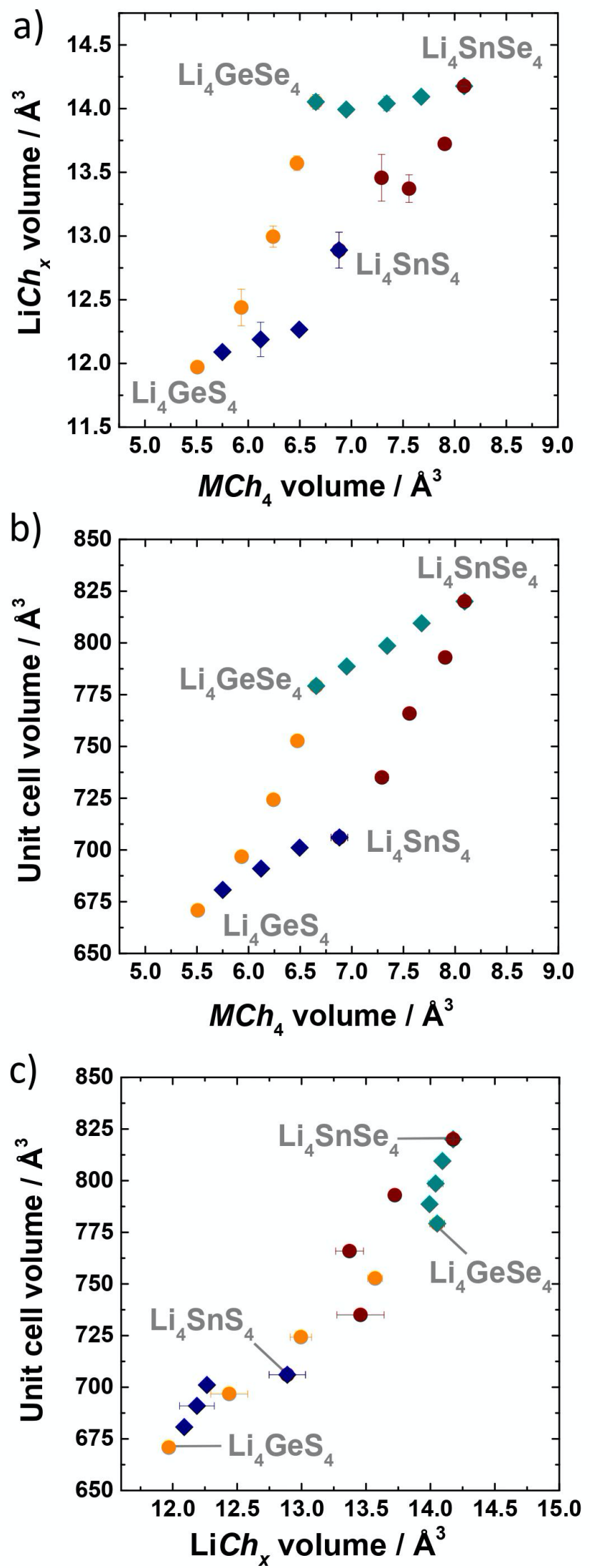

Figure 9: (a) Correlation among $\mathrm{MCh}_{4}$ and lithium polyhedral volumes. Influence of the (b) $\mathrm{MCh}_{4}$ units and (c) lithium polyhedra on the unit cell expansion. Anionic and 
cationic substitutions have different effects on the polyhedral expansion. Therefore, anionic substitutions cause major expansions of the unit cell volume by enlarging the lithium polyhedral volumes, while cationic substitutions enlarge the $\mathrm{MCh}_{4}$ volumes with smaller lattice expansion and barely affecting the lithium polyhedral pathways.

2) Volume effect on the lithium distribution and transport properties. As shown in Figure 5a, the combination of different elements that compose the crystal structure has a strong influence on the lithium distribution and disorder. As pointed out by a variety of theoretical studies, a disordered lithium substructure is of crucial importance to achieve a high ionic conductivity in the solid state. ${ }^{15,16}$ However, while the response of the lithium disorder on the transport properties is clear, the conditions to obtain this configuration are still unknown. Optimal sizes of the lithium polyhedral volumes generated by particular anion/cation combination may cause a disorder in the lithium substructure and have an impact on the transport properties. Therefore, to further investigate the influence of the lithium polyhedral size on the transport properties, in Figure $10 \mathrm{a}$ and $10 \mathrm{~b}$ the ionic conductivities and activation energies carried out from electrochemical impedance measurements are reported as a function of the average lithium polyhedral volume for all the composition along the $\mathrm{Li}_{4} \mathrm{Ge}_{1-x} \mathrm{Sn}_{x} \mathrm{~S}_{4-y} \mathrm{Se}_{y}$ solid solution series. The ionic conductivity and the activation energy describe a paraboliclike trend with the lithium polyhedral size, therefore hinting at an optimal volume for the ionic conduction.

The trends obtained from the electrical measurements are in large agreement with the dynamic process sensed by ${ }^{7} \mathrm{Li}$ NMR spin-lattice relaxation measurements (Figure 10c). In this case the activation energies extracted from the modified BPP-fits of the spin-lock NMR relaxation rates increase with the lithium polyhedral volume. The higher $E_{\mathrm{A}}$, the larger the attempt frequency determining the diffusion-induced NMR rate peaks. Even if spin-lock NMR senses only a specific dynamic process, our results support the idea that this process is linked to optimal lithium polyhedral volumes at intermediate values. The lithium polyhedral volumes of $\mathrm{Li}_{4} \mathrm{SnS}_{4}$ are close to this value, which is also reflected in the low activation energy. 
a) $\mathrm{Li}_{4} \mathrm{GeS}_{4} \quad \mathrm{Li}_{4} \mathrm{SnS}_{4} \quad \mathrm{Li}_{4} \mathrm{GeSe}_{4} \quad \mathrm{Li}_{4} \mathrm{SnSe}_{4}$

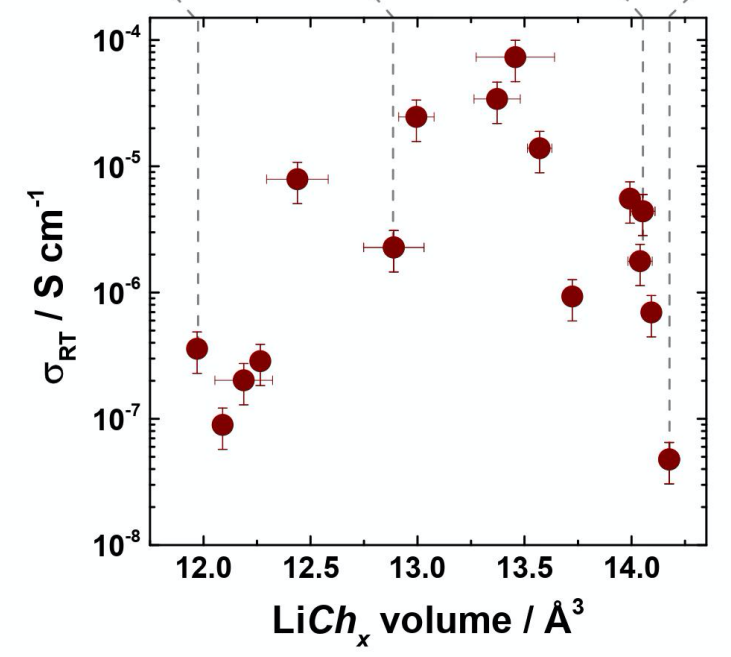

b) $\mathrm{Li}_{4} \mathrm{GeS}_{4} \quad \mathrm{Li}_{4} \mathrm{SnS}_{4} \quad \mathrm{Li}_{4} \mathrm{GeSe}_{4} \quad \mathrm{Li}_{4} \mathrm{SnSe}_{4}$

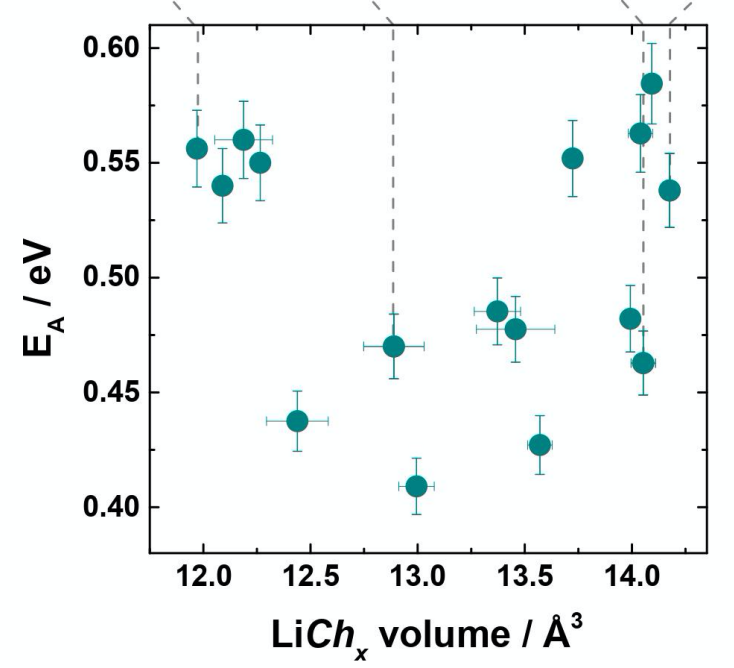

c) $\mathrm{Li}_{4} \mathrm{GeS}_{4} \quad \mathrm{Li}_{4} \mathrm{SnS}_{4} \quad \mathrm{Li}_{4} \mathrm{GeSe}_{4} \quad \mathrm{Li}_{4} \mathrm{SnSe}_{4}$

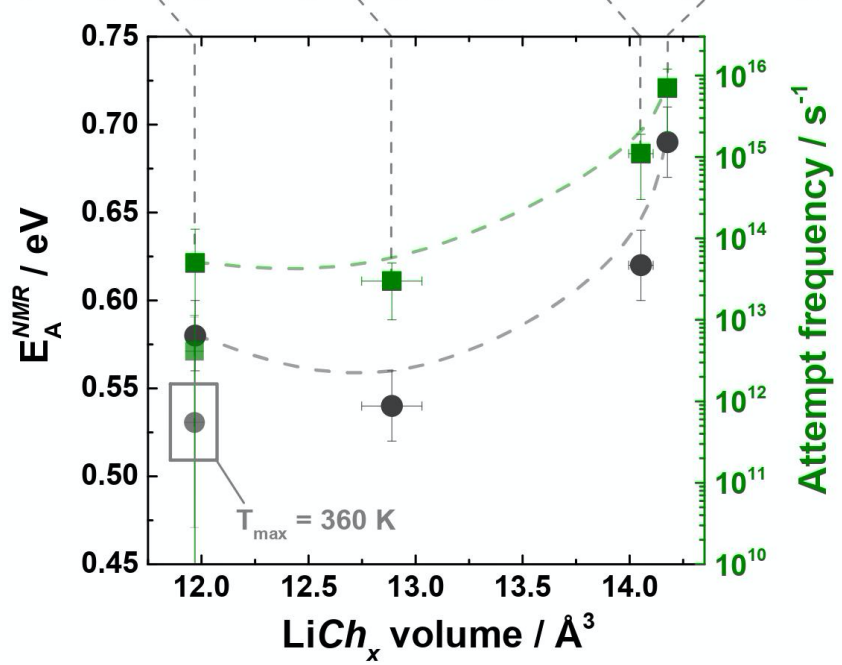

Figure 10: (a) Lithium ionic conductivity and (b) activation energy carried out from impedance spectroscopy alongside the (c) activation energies and attempt frequencies derived from the BPP-fits of the diffusion-induced ${ }^{7} \mathrm{Li} N \mathrm{NM}$ spin-lock rates as a function 
of the average lithium polyhedral volumes $\mathrm{LiCh}_{x}$. Dashed lines in panel (c) are to guide the eye; they exclude the high-T peak of $\mathrm{Li}_{4} \mathrm{GeS}_{4}$ appearing at $T_{\max }=360 \mathrm{~K}$. The results of the electrical measurements, being in line with the NMR data, reveal an optimal channel size for lithium diffusion.

While at small volumes the ionic transport is hindered due to the large energy penalty that needs to be paid when the $\mathrm{Li}^{+}$ion passes through an extremely small bottleneck, ${ }^{17}$ the exact reasons behind the decline of the ionic motion for larger volumes are still unknown. This behavior may be attributed to the fact that the mobile lithium would not migrate through the center of the structural openings but rather grazing the "walls" of the conduction pathways. This would result in larger changes of lithium coordination during diffusion that could in turn cause a higher energy penalty associated with the diffusion (Figure 11).
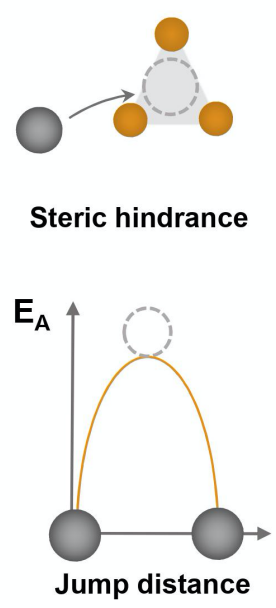

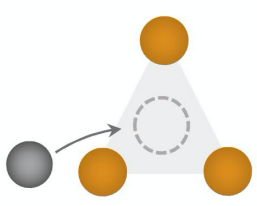

Optimal size

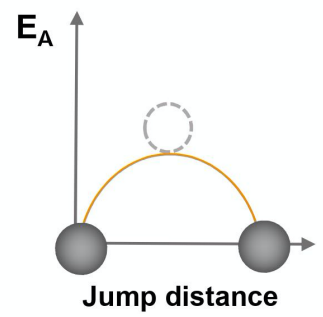

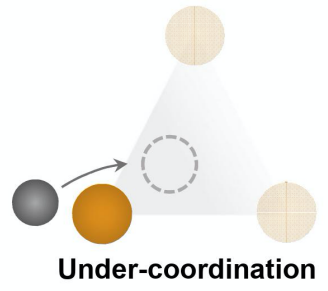

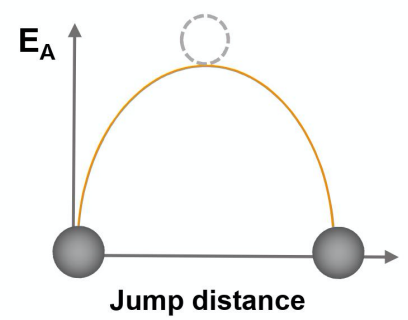

Figure 11: Illustrative representation of the lithium diffusion across a structural bottleneck and the expected energy landscape as function of the conduction pathways size. Mobile lithium ions and elements composing the structural framework are shown as gray and orange spheres, respectively.

\section{Conclusion}

In this work a systematic study of the "two-dimensional" substitution series $\mathrm{Li}_{4} \mathrm{Ge}_{1-x} \mathrm{Sn}_{x} \mathrm{~S}_{4-y} \mathrm{Se}_{y}$ has been performed to discern the influence of the different substituents on the structural modifications and transport properties. Employing Rietveld refinements against high-resolution neutron diffraction data, we show that anionic and cationic substitutions have a different impact 
on the polyhedral and unit cell volume expansion. Considering the whole substitution range, we found that the unit cell volume expands more upon anionic substitution compared to the cationic. The expansion of the unit cell is very similar to the relative expansion of the $\mathrm{LiCh}_{x}$ volume: $\sim 15 \%$ via anionic substitutions and only $\sim 5 \%$ for the cationic. This evidence is in contrast with the local evolution of the $M C h_{4}$ volumes that expand more upon cationic substitutions compared to the anionic ( $\sim 24 \%$ and $20 \%$, respectively). Therefore, performing a two-dimensional substitution series we were able to demonstrate that the $M C h_{4}$ size is not enough to explain the lattice expansion and that the lithium polyhedra play a very important role in regulating the lattice volume, while for the usually performed one-dimensional substitutions series the lattice expansion would have surely been blamed only to the size of the $M C h_{4}$ units.

Moreover, we showed that the lithium polyhedral volume has a great impact on the lithium distribution and ionic diffusion of these materials. Employing electrochemical impedance spectroscopy, we found a non-monotonic behavior of the transport properties as function of the lithium polyhedral volumes that, after an initial increase, reach a maximum and decrease for further volume expansions. This finding of an optimal lithium polyhedral volume is strongly supported by the spin-fluctuations sensed by ${ }^{7} \mathrm{Li}$ spin-lattice relaxation NMR. While the correlation between lithium polyhedral volumes and transport properties was clearly demonstrated, it is still possible that the convolution of other factors, such as the different polarizability and charge distributions of the elements that form the anionic framework, may still play a role. Ultimately this work provides a better understanding of the composition structure - transport relationships in solid electrolytes, highlighting the importance of performing two-dimensional substitution series.

\section{Acknowledgments}

The research was supported by the Deutsche Forschungsgemeinschaft (DFG) under grant number ZE 1010/4-1. This research used resources at the Spallation Neutron Source (IPTS22924.1), operated by the Oak Ridge National Laboratory. Furthermore, the authors are thankful for financial support from the FFG (Austrian Research Promotion Agency) in the frame of the competence project 'safe battery'. 


\section{Supporting Information}

Experimental neutron powder diffractograms for all explored compositions are reported, alongside with the crystallographic information files and the list of the minor secondary phases obtained by Rietveld refinements. The starting point for the refinement of all possible voids suitable for lithium occupation are also provided. Moreover, the Ge/Sn and S/Se ratios, and the extracted lattice parameters for all explored compositions are reported. In the supporting information are also tabulated the activation energies and attempt frequencies obtained by ${ }^{7} \mathrm{Li}$ $1 / T_{1 \rho}$ spin-lattice relaxation NMR carried out in the rotating frame of reference together with the temperature at which the respective peak occurs.

\section{References}

(1) Janek, J.; Zeier, W. G. A Solid Future for Battery Development. Nat. Energy 2016, 1, 16141.

(2) Famprikis, T.; Canepa, P.; Dawson, J. A.; Islam, M. S.; Masquelier, C. Fundamentals of Inorganic Solid-State Electrolytes for Batteries. Nat. Mater. 2019, 18, 1278-1291.

(3) Hu, Y. Getting Solid. Nat. Energy 2016, 1, 16042.

(4) Goodenough, J. B.; Park, K. S. The Li-Ion Rechargeable Battery: A Perspective. J. Am. Chem. Soc. 2013, 135, 1167-1176.

(5) Goodenough, J. B.; Kim, Y. Challenges for Rechargeable Li Batteries. Chem. Mater. 2010, 22, 587-603.

(6) Bielefeld, A.; Weber, D. A.; Janek, J. Modeling Effective Ionic Conductivity and Binder Influence in Composite Cathodes for All-Solid-State Batteries. ACS Appl. Mater. Interfaces 2020, 12, 12821-12833.

(7) Kraft, M. A.; Ohno, S.; Zinkevich, T.; Koerver, R.; Culver, S. P.; Fuchs, T.; Senyshyn, A.; Indris, S.; Morgan, B. J.; Zeier, W. G. Inducing High Ionic Conductivity in the Lithium Superionic Argyrodites $\mathrm{Li}_{6+}{ }_{+} \mathrm{P}_{1-\mathrm{x}} \mathrm{Ge}_{\mathrm{x}} \mathrm{S}_{5} \mathrm{I}$ for All-Solid-State Batteries. J. Am. Chem. Soc. 2018, 140, 16330-16339.

(8) Zhou, L.; Assoud, A.; Zhang, Q.; Wu, X.; Nazar, L. F. A New Family of Argyrodite Thioantimonate Lithium Superionic Conductors. J. Am. Chem. Soc. 2019, 141, 1900219013 
(9) Kato, Y.; Hori, S.; Saito, T.; Suzuki, K.; Hirayama, M.; Mitsui, A.; Yonemura, M.; Iba, H.; Kanno, R. High-Power All-Solid-State Batteries Using Sulfide Superionic Conductors. Nat. Energy 2016, 1, 16030.

(10) Liu, Z.; Fu, W.; Payzant, E. A.; Yu, X.; Wu, Z.; Dudney, N. J.; Kiggans, J.; Hong, K.; Rondinone, A. J.; Liang, C. Anomalous High Ionic Conductivity of Nanoporous $\beta$-Li $\mathrm{LS}_{3}$. J. Am. Chem. Soc. 2013, 135, 975-978.

(11) Sakuda, A.; Hayashi, A.; Tatsumisago, M. Sulfide Solid Electrolyte with Favorable Mechanical Property for All-Solid-State Lithium Battery. Sci. Rep. 2013, 3, 2261.

(12) Ohno, S.; Banik, A.; Dewald, G. F.; Kraft, M. A.; Krauskopf, T.; Minafra, N.; Till, P.; Weiss, M.; Zeier, W. G. Materials Design of Ionic Conductors for Solid State Batteries. Prog. Energy 2020, 2, 022001.

(13) Culver, S. P.; Koerver, R.; Krauskopf, T.; Zeier, W. G. Designing Ionic Conductors: The Interplay between Structural Phenomena and Interfaces in Thiophosphate-Based SolidState Batteries. Chem. Mater. 2018, 30, 4179-4192.

(14) Bachman, J. C.; Muy, S.; Grimaud, A.; Chang, H.; Pour, N.; Lux, S. F.; Paschos, O.; Maglia, F.; Lupart, S.; Lamp, P.; et al. Inorganic Solid-State Electrolytes for Lithium Batteries : Mechanisms and Properties Governing Ion Conduction. Chem. Rev 2016, 116, $140-162$.

(15) He, X.; Bai, Q.; Liu, Y.; Nolan, A. M.; Ling, C.; Mo, Y. Crystal Structural Framework of Lithium Super-Ionic Conductors. Adv. Energy Mater. 2019, 9, 1902078.

(16) He, X.; Zhu, Y.; Mo, Y. Origin of Fast Ion Diffusion in Super-Ionic Conductors. Nat. Commun. 2017, 8, 15893.

(17) Wang, Y.; Richards, W. D.; Ong, S. P.; Miara, L. J.; Kim, J. C.; Mo, Y.; Ceder, G. Design Principles for Solid-State Superionic Conductors. Nat. Mater. 2015, 14, 1026.

(18) Xu, Z.; Zhu, H. Anion Charge and Lattice Volume Maps for Searching Lithium Superionic Conductors. Chem. Mater. 2020, 32, 4618-4626.

(19) Xu, Z.; Chen, X.; Chen, R.; Li, X.; Zhu, H. Anion Charge and Lattice Volume Dependent Lithium Ion Migration in Compounds with Fcc Anion Sublattices. npj Comput. Mater. 2020, 6, Articel number: 47.

(20) Rodger, A. R.; Kuwano, J.; West, A. R. $\mathrm{Li}^{+}$Ion Conducting $\gamma$ Solid Solutions in the Systems $\mathrm{Li}_{4} \mathrm{XO}_{4}-\mathrm{Li}_{3} \mathrm{YO}_{4}: \mathrm{X}=\mathrm{Si}, \mathrm{Ge}, \mathrm{Ti} ; \mathrm{Y}=\mathrm{P}, \mathrm{As}, \mathrm{V}$; Li4 $\mathrm{XO}_{4}-\mathrm{LiZO}_{2}: \mathrm{Z}=\mathrm{Al}, \mathrm{Ga}, \mathrm{Cr}$ 
and $\mathrm{Li}_{4} \mathrm{GeO}_{4}-\mathrm{Li}_{2} \mathrm{CaGeO}_{4}$. Solid State Ionics 1985, 15, 185-198.

(21) Ong, S. P.; Mo, Y.; Richards, D.; Miara, L.; Lee, H. S.; Ceder, G. Phase Stability, Electrochemical Stability and Ionic Conductivity of the $\mathrm{Li}_{10 \pm 1} \mathrm{MP}_{2} \mathrm{X}_{12}(\mathrm{M}=\mathrm{Ge}, \mathrm{Si}, \mathrm{Sn}$, $\mathrm{Al}$ or $\mathrm{P}$, and $\mathrm{X}=\mathrm{O}, \mathrm{S}$ or $\mathrm{Se}$ ) Family of Superionic Conductors. Energy Environ. Sci. 2013, 6, 148-156.

(22) Martınez-Juarez, A.; Pecharroma, C.; Iglesias, J. E.; Rojo, J. M. Relationship between Activation Energy and Bottleneck Size for $\mathrm{Li}^{+}$Ion Conduction in NASICON Materials of Composition LiMM'(PO 4$)_{3} ; \mathrm{M}, \mathrm{M}^{\prime}=\mathrm{Ge}, \mathrm{Ti}, \mathrm{Sn}, \mathrm{Hf}$. J. Phys. Chem. B 1998, 102, 372 375.

(23) Aono, H.; Imanaka, N.; Adachi, G. High $\mathrm{Li}^{+}$Conducting Ceramics. Acc. Chem. Res. 1994, 27, 265-270.

(24) Krauskopf, T.; Culver, S. P.; Zeier, W. G. Bottleneck of Diffusion and Inductive Effects in $\mathrm{Li}_{10} \mathrm{Ge}_{1-\mathrm{x}} \mathrm{Sn}_{\mathrm{x}} \mathrm{P}_{2} \mathrm{~S}_{12}$. Chem. Mater. 2018, 30, 1791-1798.

(25) Kraft, M. A.; Culver, S. P.; Calderon, M.; Bocher, F.; Krauskopf, T.; Senyshyn, A.; Dietrich, C.; Zevalkink, A.; Janek, J.; Zeier, W. G. Influence of Lattice Polarizability on the Ionic Conductivity in the Lithium Superionic Argyrodites $\mathrm{Li}_{6} \mathrm{PS}_{5} \mathrm{X}(\mathrm{X}=\mathrm{Cl}, \mathrm{Br}, \mathrm{I}) . J$. Am. Chem. Soc. 2017, 139, 10909-10918.

(26) Krauskopf, T.; Muy, S.; Culver, S. P.; Ohno, S.; Delaire, O.; Shao-horn, Y.; Zeier, W. G. Comparing the Descriptors for Investigating the Influence of Lattice Dynamics on Ionic Transport Using the Superionic Conductor. J. Am. Chem. Soc. 2018, 140, 1446414473.

(27) Krauskopf, T.; Pompe, C.; Kraft, M. A.; Zeier, W. G. Influence of Lattice Dynamics on $\mathrm{Na}^{+}$Transport in the Solid Electrolyte $\mathrm{Na}_{3} \mathrm{PS}_{4-\mathrm{x}} \mathrm{Se}_{\mathrm{x}}$. Chem. Mater. 2017, 29, 8859-8869.

(28) Muy, S.; Bachman, J. C.; Giordano, L.; Chang, H.; Abernathy, D. L.; Bansal, D.; Delaire, O.; Hori, S.; Kanno, R.; Maglia, F.; et al. Tuning Mobility and Stability of Lithium Ion Conductors Based on Lattice Dynamics. Energy Environ. Sci. 2018, 11, 850-859.

(29) West, A. R. Inorganic Functional Materials: Optimization of Properties by Structural and Compositional Control. Chem. Rec. 2006, 6, 206-216.

(30) Kanno, R.; Hata, T.; Kawamoto, Y.; Irie, M. Synthesis of a New Lithium Ionic Conductor, Thio-LISICON-Lithium Germanium Sulfide System. Solid State Ionics 2000, 130, 97-104. 
(31) Homma, K.; Yonemura, M.; Kobayashi, T.; Nagao, M.; Hirayama, M.; Kanno, R. Crystal Structure and Phase Transitions of the Lithium Ionic Conductor $\mathrm{Li}_{3} \mathrm{PS}_{4}$. Solid State Ionics 2011, 182, 53-58.

(32) Kaup, K.; Zhou, L.; Huq, A.; Nazar Linda F. Impact of the Li Substructure on the Diffusion Pathways in Alpha and Beta $\mathrm{Li}_{3} \mathrm{PS}_{4}$ : An in-Situ High Temperature Neutron Diffraction Study. J. Mater. Chem. A 2020, Accepted Manuscript.

(33) Ahn, B.; Huggins, R. A. Synthesis and Lithium Conductivities of $\mathrm{Li}_{2} \mathrm{SiS}_{3}$ and $\mathrm{Li}_{4} \mathrm{SiS}_{4}$. Mat. Res. Bull. 1989, 24, 889-897.

(34) Minafra, N.; Culver, S. P.; Li, C.; Senyshyn, A.; Zeier, W. G. Influence of the Lithium Substructure on the Diffusion Pathways and Transport Properties of the Thio-LISICON $\mathrm{Li}_{4} \mathrm{Ge}_{1-\mathrm{x}} \mathrm{Sn}_{\mathrm{x}} \mathrm{S}_{4}$. Chem. Mater. 2019, 31, 3794-3802.

(35) Kaib, T.; Haddadpour, S.; Kapitein, M.; Bron, P.; Schröder, C.; Eckert, H.; Roling, B.; Dehnen, S. New Lithium Chalcogenidotetrelates, LiChT: Synthesis and Characterization of the $\mathrm{Li}^{+}$-Conducting Tetralithium Ortho-Sulfidostannate $\mathrm{Li}_{4} \mathrm{SnS}_{4}$. Chem. Mater. 2012 , 24, 2211-2219.

(36) Murayama, M.; Kanno, R.; Kawamoto, Y.; Kamiyama, T. Structure of the ThioLISICON, Li4 $\mathrm{GeS}_{4}$. Solid State Ionics 2002, 154-155, 789-794.

(37) Matsushita, Y.; Kanatzidis, M. G. Synthesis and Structure of $\mathrm{Li}_{4} \mathrm{GeS}_{4}$. Zeitschrift für Naturforsch. B 1998, 53, 23-30.

(38) Macneil, J. H.; Massi, D. M.; Zhang, J. H.; Rosmus, K. A.; Brunetta, C. D.; Gentile, T. A.; Aitken, J. A. Synthesis, Structure, Physicochemical Characterization and Electronic Structure of Thio-Lithium Super Ionic Conductors, $\mathrm{Li}_{4} \mathrm{GeS}_{4}$ and $\mathrm{Li}_{4} \mathrm{SnS}_{4}$. J. Alloys Compd. 2014, 586, 736-744.

(39) Murayama, M.; Kanno, R.; Irie, M.; Ito, S.; Hata, T.; Sonoyama, N.; Kawamoto, Y. Synthesis of New Lithium Ionic Conductor Thio-LISICON - Lithium Silicon Sulfides System. J. Solid State Chem. 2002, 168, 140-148.

(40) Murayama, M.; Sonoyama, N.; Yamada, A.; Kanno, R. Material Design of New Lithium Ionic Conductor, Thio-LISICON, in the $\mathrm{Li}_{2} \mathrm{~S}_{-} \mathrm{P}_{2} \mathrm{~S}_{5}$ System. Solid State Ionics 2004, 170, $173-180$.

(41) Leube, B. T.; Inglis, K. K.; Carrington, E.; Sharp, P. M.; Felix, J.; Neale, A. R.; Manning, T. D.; Pitcher, M. J.; Hardwick, L. J.; Dyer, M. S.; et al. Lithium Transport in LiMM'S 
$\left(\mathrm{M}=\mathrm{Al}, \mathrm{Ga}\right.$ and $\left.\mathrm{M}^{\prime}=\mathrm{Ge}, \mathrm{Sn}\right)$ : Combined Crystallographic, Conductivity, Solid State NMR and Computational Studies. Chem. Mater. 2018, 30, 7183-7200.

(42) Kanno, R.; Murayama, M. Lithium Ionic Conductor Thio-LISICON: The $\mathrm{Li}_{2} \mathrm{~S}-\mathrm{GeS}_{2}-$ $\mathrm{P}_{2} \mathrm{~S}_{5}$ System. J. Electrochem. Soc. 2001, 148, A742-A746.

(43) Zhou, L.; Assoud, A.; Shyamsunder, A.; Huq, A.; Zhang, Q.; Hartmann, P.; Kulisch, J.; Nazar, L. F. An Entropically Stabilized Fast-Ion Conductor: $\mathrm{Li}_{3.25}\left[\mathrm{Si}_{0.25} \mathrm{P}_{0.75}\right] \mathrm{S}_{4}$. Chem. Mater. 2019, 31, 7801-7811.

(44) Sahu, G.; Rangasamy, E.; Li, J.; Chen, Y.; An, K.; Dudney, N.; Liang, C. A HighConduction Ge Substituted $\mathrm{Li}_{3} \mathrm{AsS}_{4}$ Solid Electrolyte with Exceptional Low Activation Energy. J. Mater. Chem. A 2014, 2, 1039-10403.

(45) Sahu, G.; Lin, Z.; Li, J.; Liu, Z.; Dudney, N.; Liang, C. Air-Stable, High-Conduction Solid Electrolytes of Arsenic-Substituted $\mathrm{Li}_{4} \mathrm{SnS}_{4}$. Energy Environ. Sci. 2014, 7, $1053-$ 1058 .

(46) Zhang, Z.; Zhang, J.; Sun, Y.; Jia, H.; Peng, L.; Zhang, Y.; Xie, J. Li4-x $\mathrm{Sb}_{\mathrm{x}} \mathrm{Sn}_{1-\mathrm{x}} \mathrm{S}_{4}$ Solid Solutions for Air-Stable Solid Electrolytes. J. Energy Chem. 2020, 41, 171-176.

(47) Zhang, Z.; Li, H.; Kaup, K.; Zhou, L.; Roy, P.-N.; Linda, F. N. Targeting Superionic Conductivity by Turning on Anion Rotation at Room Temperature in Fast Ion Conductors. Matter 2020, 2, 1667-1684.

(48) Kamaya, N.; Homma, K.; Yamakawa, Y.; Hirayama, M.; Kanno, R.; Yonemura, M.; Kamiyama, T.; Kato, Y.; Hama, S.; Kawamoto, K.; et al. A Lithium Superionic Conductor. Nat. Mater. 2011, 10, 682-686.

(49) Neveu, A.; Pel, V.; Jordy, C.; Pralong, V. Exploration of Li-P-S-O Composition for Solid-State Electrolyte Materials Discovery. J. Power Sources 2020, 467, 228250.

(50) Suzuki, K.; Sakuma, M.; Hori, S.; Nakazawa, T.; Nagao, M.; Yonemura, M.; Hirayama, M.; Kanno, R. Synthesis, Structure, and Electrochemical Properties of Crystalline Li-PS-O Solid Electrolytes: Novel Lithium-Conducting Oxysulfides of $\mathrm{Li}_{10} \mathrm{GeP}_{2} \mathrm{~S}_{12}$ Family. Solid State Ionics 2016, 288, 229-234.

(51) Wang, X.; Xiao, R.; Li, H.; Chen, L. Oxygen-Driven Transition from Two-Dimensional to Three-Dimensional Transport Behaviour in $\beta-\mathrm{Li}_{3} \mathrm{PS}_{4}$ Electrolyte. Phys. Chem. Chem. Phys. 2016, 18, 21269-21277.

(52) De Klerk, N. J. J.; Maas, E. Van Der; Wagemaker, M. Analysis of Diffusion in Solid- 
State Electrolytes through MD Simulations, Improvement of the Li-Ion Conductivity in $\beta-\mathrm{Li}_{3} \mathrm{PS}_{4}$ as an Example. ACS Appl. Energy Mater. 2018, 1, 3230-3242.

(53) Kaib, T.; Bron, P.; Haddadpour, S.; Mayrhofer, L.; Pastewka, L.; Ja, T. T.; Moseler, M.; Roling, B.; Dehnen, S. Lithium Chalcogenidotetrelates: LiChT-Synthesis and Characterization of New $\mathrm{Li}^{+}$Ion Conducting Li/Sn/Se Compounds. Chem. Mater. 2013, 25, 2961-2969.

(54) Huq, A.; Kirkham, M.; Peterson, P. F.; Hodges, J. P.; Pamela, S.; Page, K.; Hu, T.; Iverson, E. B.; Rennich, G. POWGEN : Rebuild of a Third-Generation Powder Diffractometer at the Spallation Neutron Source Research Papers. J. Appl. Crystallogr. 2019, 52, 1189- 876

(55) Coelho, A. A. TOPAS-Academic 2007, Brisbane, Australia.

(56) Roeder, S.; Fukushima, E. Experimental Pulse NMR: A Nuts and Bolts Approach 1981.

(57) Shannon, R. D. Revised Effective Ionic Radii in Halides and Chalcogenides. Acta Cryst. 1976, A32, 751-767.

(58) Deng, Y.; Eames, C.; Fleutot, B.; Chotard, J.; Suard, E.; Masquelier, C.; Islam, M. S. Enhancing the Lithium Ion Conductivity in Lithium Superionic Conductor (LISICON) Solid Electrolytes through a Mixed Polyanion Effect. ACS Appl. Mater. Interfaces 2017, 9, 7050-7058.

(59) Irvine, B. J. T. S.; Sinclair, D. C.; West, A. R. Electroceramics: Characterization by Impedance Spectroscopy. Adv. Mater. 1990, 2, 132-138.

(60) Ohno, S.; Bernges, T.; Buchheim, J.; Duchardt, M.; Hatz, A.-K.; Kraft, M. A.; Kwak, H.; Santhosha, A. L.; Liu, Z.; Minafra, N.; et al. How Certain Are the Reported Ionic Conductivities of Thiophosphate-Based Solid Electrolytes? An Interlaboratory Study. ACS Energy Lett. 2020, 5, 910-915.

(61) Wilkening, M.; Heitjans, P. From Micro to Macro: Access to Long-Range $\mathrm{Li}^{+}$Diffusion Parameters in Solids via Microscopic ${ }^{6,7} \mathrm{Li}$ Spin-Alignment Echo NMR Spectroscopy. ChemPhysChem 2012, 13, 53-65.

(62) Bloembergen, N.; Purcell, E. M.; Pound, R. V. Relaxation Effects in Nuclear Magnetic Resonance Absorption. Phys. Rev. 1948, 73, 679.

(63) Kuhn, A.; Narayanan, S.; Spencer, L.; Goward, G.; Thangadurai, V.; Wilkening, M. Li Self-Diffusion in Garnet-Type $\mathrm{Li}_{7} \mathrm{La}_{3} \mathrm{Zr}_{2} \mathrm{O}_{12}$ as Probed Directly by Diffusion-Induced Li 
Spin-Lattice Relaxation NMR Spectroscopy. Phys. Rev. B 2011, 83, 094302.

(64) Wilkening, M.; Bork, D.; Indris, S.; Heitjans, P. Diffusion in Amorphous $\mathrm{LiNbO}_{3}$ Studied by ${ }^{7}$ Li NMR-Comparison with the Nano-and Microcrystalline Material. Phys. Chem. Chem. Phys. 2002, 4, 3246-3251.

(65) Culver, S.; Squires, A.; Minafra, N.; Armstrong, C.; Krauskopf, T.; Boecher, F.; Li, C.; Morgan, J. B.; Zeier, G. W. Evidence for a Solid-Electrolyte Inductive Effect in Superionic Conductors. ChemRxiv. 2020, Preprint. DOI:10.26434/chemrxiv.13075745.v1 
For table of contents only

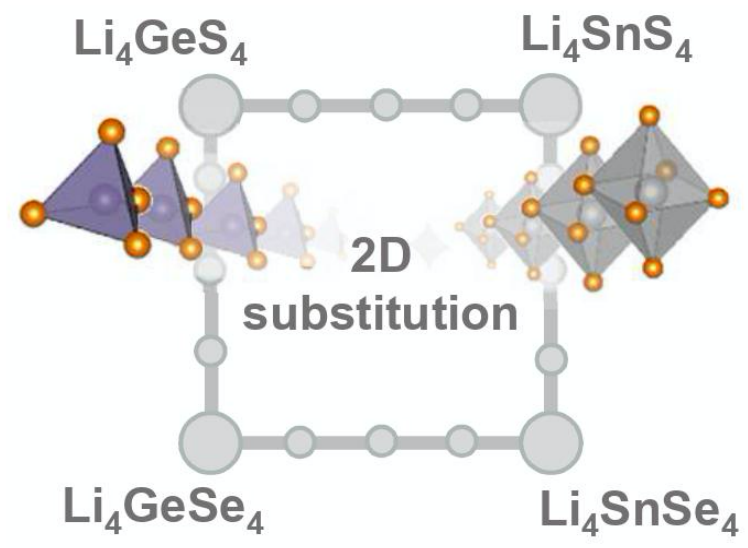

